\title{
CONDITIONAL GAUGE AND POTENTIAL THEORY FOR THE SCHRÖDINGER OPERATOR
}

\author{
M. CRANSTON, E. FABES AND Z. ZHAO
}

\begin{abstract}
This paper extends the Conditional Gauge Theorem to more general operators and less regular domains than in previous works. We use this to obtain potential-theoretic results for the Schrödinger equation.
\end{abstract}

1. Introduction. We study the Schrödinger operator $L=-A+q$ on a domain $D$. Here $A$ is a second-order elliptic divergence form operator, $q$ is a function in the Kato class and $D$ is a bounded Lipschitz subdomain of $R^{d}, d \geq 3$. The case $d=2$ will be treated in a subsequent note.

Our main results are first to prove a conditional gauge theorem in this context and second to extend potential-theoretic results valid for $A$ to $L$ using the Conditional Gauge Theorem.

In order to describe the conditional gauge, introduce the measure $P_{y}^{x}$ for the diffusion $X$ with infinitesimal generator $A$ started at $x \in \bar{D}$ but conditioned to converge to $y \in \bar{D}$ at the path lifetime $\tau_{D}$. Set

$$
e_{q}\left(\tau_{D}\right)=\exp \left\{-\int_{0}^{\tau_{D}} q(X(s)) d s\right\} .
$$

Then the conditional gauge is defined as

$$
F(x, y)=E_{y}^{x} e_{q}\left(\tau_{D}\right) .
$$

The Conditional Gauge Theorem (4.2) asserts that the finiteness of $F(x, y)$ at some point $\left(x_{0}, y_{0}\right) \in \bar{D} \times \bar{D}$ with $x_{0} \neq y_{0}$ implies the existence of positive constants $c_{1}$ and $c_{2}$ such that $c_{1} \leq F(x, y) \leq c_{2}$ for all $(x, y) \in \bar{D} \times \bar{D}$. This has been established in various combinations of different situations than described here, usually with $x_{0} \in D, y_{0} \in \partial D$, always with $A$ replaced by $\Delta / 2$, sometimes with bounded $q$ or $q \in L^{p}(D)$ for some $p>d / 2$ and a class of domains called rapidly exhaustible [20], or when $q$ is in the Kato class $D$ was assumed to have $C^{1,1}$ boundary [31]. The main difficulty we have overcome is in relaxing the smoothness assumptions on $D$ when $q$ is in the Kato class. Replacing $\Delta / 2$ with $A$ comes for free given the results of $[8$ and 23]. A list of results on the conditional gauge include $[\mathbf{7}, 10,19,20$, $\mathbf{2 8}, \mathbf{3 0}]$. Our proof of Theorem 4.2 follows techniques developed and refined in [29, 30 and 10]. These techniques rely on a certain Green function estimate (Theorem

Received by the editors April 21, 1986 and, in revised form, March 16, 1987.

1980 Mathematics Subject Classification (1985 Revision). Primary 60J45; Secondary 31B25.

This research was completed while the authors were visiting the Institute for Mathematics and its Applications. We would like to express our thanks for the hospitality of the Institute.

Research of the first author partially supported by NSF Grant DMS 85-3332.

Research of the second author partially supported by NSF Grant DMS 84-21377. 
3.1) which follows from the boundary Harnack principle for $A$ on $D$ and various estimates on the Green function for $A$ on $D$.

The potential-theoretic results which we obtain for $L$ follow from two simple identities involving the conditional gauge. Let $w_{L}^{x}$ and $w^{x}$ be the harmonic measures for $L$ and $-A$, respectively, evaluated at $x$. Correspondingly, let $G_{L}$ and $G$ be the Green functions for $L$ and $-A$. Then, assuming $F(\cdot, \cdot) \not \equiv \infty$, we have (Theorem 4.8) $w_{L}^{x}(d z)=F(x, z) w^{x}(d z)$ and $G_{L}(x, y)=F(x, y) G(x, y)$. These identities allow the transfer to $L$ of potential-theoretic results valid for $A$. $\S 5$ is composed of such results for $L$, e.g. Harnack's principle, boundary Harnack principle, Martin representation, etc., all under the assumption $F\left(x_{0}, y_{0}\right)<\infty$ for some $\left(x_{0}, y_{0}\right) \in \bar{D} \times \bar{D}, x_{0} \neq y_{0}$. Some of these are not new; Harnack's principle was established in [1] for $A=\Delta / 2$ and using different methods in [9] in the generality in which it appears here. In [6] some of our results were obtained using the theory of harmonic spaces. Many of our results, however, appear to be new.

$\S 4$ contains the so-called Gauge Theorem, due to Chung and Rao [12] for $A=$ $\Delta / 2$, and the Conditional Gauge Theorem. We also prove here the joint continuity of $F(x, y)$ on $\bar{D} \times \bar{D}$. This is related to the work of [7] where $A=\Delta / 2$ and $D$ is a ball.

$\S 3$ provides the necessary Green function estimates for the proof of the Conditional Gauge Theorem.

$\S 2$ discusses preliminaries and notation.

\section{Preliminaries.}

2.1. Assumptions on $D, A$ and $q$. Throughout this work we make the following assumptions on $D, A$ and $q$. The domain $D \subseteq R^{d}$ will be bounded and Lipschitz. That $D$ is Lipschitz means there exist a pair of positive numbers $r_{0}$ and $M$ such that for every $z \in \partial D$, local coordinates can be selected so that $\Delta\left(z, r_{0}\right)=B\left(z, r_{0}\right) \cap \partial D$ is the graph of a Lipschitz function $\phi$ with $|\nabla \phi| \leq M$. The constants $r_{0}$ and $M$ determine what will be called the Lipschitz character of $D$ and any constants depending on the Lipschitz character of $D$ will depend on $D$ through $r_{0}$ and $M$.

The operator $A$ will be a uniformly elliptic, divergence form operator with bounded measurable coefficients. That is

$$
A=\sum_{i, j=1}^{d} \frac{\partial}{\partial x^{i}}\left(a_{i j}(x) \frac{\partial}{\partial x^{j}}\right)
$$

with $a_{i j} \in L^{\infty}(D, m), m$ is Lebesgue measure, such that $a_{i j}=a_{j i}$. In addition, there is a positive number $\lambda$ so that for any $x \in D, \xi \in R^{d}$ we have

$$
\lambda^{-1}|\xi|^{2} \leq \sum_{i, j=1}^{d} a_{i j}(x) \xi_{i} \xi_{j} \leq \lambda|\xi|^{2} .
$$

The class of such operators $A$ will be denoted by $\mathcal{E}(\lambda)$, the dependence on $D$ being understood.

We consider functions $q \in K_{d}(D)$, the Kato class, defined by either of the two equivalent conditions, for $d \geq 3$ :

$$
\lim _{r \downarrow 0} \sup _{x \in D} \int_{|x-y| \leq r} \frac{|q|(y)}{|x-y|^{d-2}} d y=0
$$


or

$$
\lim _{t \downarrow 0} \sup _{x \in D} E^{x}\left[\int_{0}^{t} 1_{D} \cdot|q|(X(s)) d s\right]=0,
$$

where $\left(X, P^{x}\right)$ is the diffusion with generator $A$. When $d=2,|x-y|^{2-d}$ is replaced by $-\ln |x-y|$ in (2.1.1).

2.2. Convention on constants. We adopt the convention that constants may change their value but not their dependence from one use to the next. More importantly, unless specified otherwise, all constants depend on $\lambda, r_{0}, M$ and $q$.

When two functions $\phi$ and $\psi$ are defined on a set $S$ we write $\phi \simeq \psi$ when a two-sided inequality $c_{1} \phi \leq \psi \leq c_{2} \phi$ holds on $S$ for two positive constants $c_{1}$ and $c_{2}$.

2.3. Potential theory for $A$. Our results depend on the behavior of positive solutions to the equation $A u=0$ on $D$. We denote this class by $A^{+}$and the class of positive solutions to $L u=0$ on $D$ by $\mathcal{L}^{+}$. By solutions we mean in the weak sense. However, Chiarenza et al. [9] showed elements of $\mathcal{L}^{+}$are continuous on $D$ with a modulus of continuity depending on $q$. Functions $u \in A^{+}$are locally Hölder continuous [16 and 25]. Such results as the boundary Harnack principle for $A^{+}$will be assumed known to the reader. Those not familiar with this principle may look at Theorem 5.2. Those interested in the proof of the boundary Harnack principle for $A^{+}$should see [8]. The Harnack chain arguments which we employ may be found in [22] for $A=\Delta / 2$ and remain valid for $A \in \mathcal{E}(\lambda)$.

An important consequence of the boundary Harnack and weak maximum principles is the existence of a Hölder continuous extension of $u / v$ to $B(z, r) \cap \bar{D}$ when $u, v \in A^{+}$vanish continuously on $B(z, 2 r) \cap \partial D, z \in \partial D$. An easy to read argument for this is given in [2].

We freely use bounds for the Green function of $A$ on $D$ established in [23]. Among those used are

$$
\begin{gathered}
G(x, y) \leq c|x-y|^{2-d}, \\
G(x, y) \geq c_{\alpha}|x-y|^{2-d}, \quad \text { whenever }|x-y| \leq \alpha \delta(x),
\end{gathered}
$$

where $0<\alpha<1$ and $c_{\alpha}$ depends on $\alpha, \lambda, M$ and $r_{0}, \delta(x)=\operatorname{dist}(x, \partial D)$,

$$
\int_{D} G(x, y) d y \leq c(m(D))^{2 / d}
$$

In order to avoid ambiguity we now describe what we refer to as Carleson's estimate.

Let $u \in A^{+}$be such that $u$ vanishes on $B(z, 2 r) \cap \partial D$ for some $z \in \partial D, r<r_{0}$. Then there is a constant $c$ depending only on the Lipschitz character of $D$ such that for any point $A$ with $|A-z| \leq r$, $\operatorname{dist}(A, \partial D)>r / 2$, we have $u(x) \leq c u(A), x \in B(z, r) \cap D$.

2.4. The diffusion and conditional diffusion. Let $\left(X_{t}, P^{x}\right)$ be the diffusion with infinitesimal generator $A$ killed on exiting $D$. This diffusion is discussed, among other places, in [4]. As remarked there the Dirichlet form associated to $A$ is regular according to [21], and the theory of Dirichlet forms can be used to construct this process. 
According to $[\mathbf{3}]$, there is a unique positive Green function for the equation $A=\partial / \partial t$ on $D \times(0, \infty)$, i.e. a solution to the problem

$$
\begin{aligned}
A p(t, x, \cdot) & =\frac{\partial}{\partial t} p(t, x, \cdot), \quad t>0, x \in D, \\
p(t, x, z) & =0, \quad t>0, \quad z \in \partial D, \\
\lim _{t \downarrow 0} p(t, x, y) & =\delta_{x}(d y), \quad x, y \in D .
\end{aligned}
$$

This acts as the subprobability transition density for $X$, denoted by

$$
P^{x}(X(t) \in d y)=p(t, x, y) d y \text {. }
$$

We will take $\left(X, P^{x}\right)$ to be defined on a space of paths $\left(\Omega, F_{t}, \theta_{t}\right)$ where $\Omega=$ $\left\{\omega: \exists t_{\omega} \in(0, \infty]\right.$ s.t. $\omega:\left[0, t_{\omega}\right) \rightarrow D$ is continuous, $\left.\omega(t)=\partial, t \geq t_{\omega}\right\}$, where $\partial$ is an isolated point adjoined to $D$.

The $\sigma$-fields $F_{t}=\sigma(X(s, \omega), 0 \leq s \leq t)$, where $X(s, \omega)=\omega(s)$, are the natural ones and $\theta_{t}$ is the shift, $\theta_{t} \omega(\cdot)=\omega(t+\cdot)$. Define for $B \subseteq D, \tau_{B}=\inf \{t>0: X(t) \notin$ $B$. Then $\tau_{D}(\omega)=t_{\omega}$ and at the time $\tau_{D}, X$ goes to $\partial$ and remains there. By (2.3.1),

$$
E^{x} \tau_{D}=\int_{D} G(x, y) d y \leq c \int_{D}|x-y|^{2-d} d y<\infty
$$

so $P^{x}\left(\tau_{D}<\infty\right)=1$. Also, $X\left(\tau_{D^{-}}\right)=\lim _{t \dagger \tau_{D}} X(t)$ exists $P^{x}$ a.s. since $\lim _{t \uparrow \tau_{D}} X(t)$ exists in the Martin topology and by [8] the Martin boundary for $A$ on $D$ is the Euclidean boundary and the Martin topology is the Euclidean one. The Martin kernel, according to [8], is given by

$$
K(x, z)=\lim _{y \rightarrow z} G\left(\tilde{x}_{0}, y\right)^{-1} G(x, y) \quad \text { for } z \in \partial D
$$

where $\tilde{x}_{0} \in D$ is fixed.

A central role is played by the diffusion conditioned on its terminal point as introduced in [17]. Namely, the subprobability transition density for $X$ conditioned on $X\left(\tau_{D^{-}}\right)=z$ is

$$
p^{z}(t, x, y)=K(x, z)^{-1} p(t, x, y) K(y, z) .
$$

A measure $P_{z}^{x}$ can be constructed on $\Omega$ such that

$$
P_{z}^{x}(X(0)=x)=1 \quad \text { and } \quad P_{z}^{x}(X(t) \in d y)=p^{z}(t, x, y) d y
$$

with the additional properties

$$
P_{z}^{x}\left(X\left(\tau_{D^{-}}\right)=z\right)=1 \quad \text { and } \quad P_{z}^{x}(\cdot)=P^{x}\left(\cdot \mid X\left(\tau_{D^{-}}\right)=z\right) .
$$

For computations we often employ the formula

$$
P_{z}^{x}\left(\Lambda \cap\left\{s<\tau_{D}\right\}\right)=K(x, z)^{-1} E^{x}\left(K(X(s), z) ; \Lambda \cap\left\{s<\tau_{D}\right\}\right), \quad \Lambda \in F_{s} .
$$

The Green function corresponding to $\left(X, P_{z}^{x}\right)$ is

$$
G^{z}(x, y)=\int_{0}^{\infty} p^{z}(t, x, y) d t=K(x, z)^{-1} G(x, y) K(y, z)
$$

and the interpretation $E_{z}^{x} \tau_{D}=\int_{D} G^{z}(x, y) d y$ remains valid. A consequence of Theorem 3.1 is that $E_{z}^{x} \tau_{D}<\infty$. 
The diffusion $\left(X, P^{x}\right)$ can also be conditioned to converge to a point $y \in D$ at the path lifetime $\tau_{D}=\inf \{t>0: X(t) \notin D\}$. This conditioned diffusion has transition density

$$
p^{y}(t, x, w)=G(x, y)^{-1} p(t, x, w) G(w, y)
$$

with Green function

$$
G^{y}(x, w)=G(x, y)^{-1} G(x, w) G(w, y) .
$$

The corresponding measure on paths is still denoted by $P_{y}^{x}$. We interpret $P_{y}^{y}$ as the unit point mass on the path $\omega=\omega_{y}$ with $\omega_{y}(0)=y, \omega_{y}(s)=\partial$ for $s>0$. Once again we have

$$
P_{y}^{x}\left(\Lambda \cap\left\{s<\tau_{D}\right\}\right)=G(x, y)^{-1} E^{x}\left[G(X(s), y) ; \Lambda \cap\left\{s<\tau_{D}\right\}\right], \quad \Lambda \in F_{s},
$$

and

$$
E_{y}^{x} \tau_{D}=\int_{D} G^{y}(x, w) d w
$$

Up to now, the conditioned processes have always begun inside $D$. In [17], it was noticed, for Brownian motion, that the diffusion can be started at a boundary point $z$, when conditioned to converge to a point $y \in D$. In [26] a nice example was presented of a bounded domain $D \subseteq R^{2}$ and $A=\Delta / 2$, which has distinct minimal Martin boundary points $z_{1}$ and $z_{2}$ for which there is no measure on paths $P_{z_{2}}^{z_{1}}$. More specifically, there is no measure satisfying the prescriptions:

$$
\begin{aligned}
& P_{z_{2}}^{z_{1}}\left(X(0)=z_{1}\right)=1, \\
& P_{z_{2}}^{z_{1}}(X(t+s) \in d y \mid X(s)=x)=p^{z_{2}}(t, x, y) d y, \\
& P_{z_{2}}^{z_{1}}\left(X\left(\tau_{D^{-}}\right)=z_{2}\right)=1 .
\end{aligned}
$$

However, when $A \in \mathcal{E}(\lambda)$ and $D$ is a bounded Lipschitz domain such measures exist. This result is due to [26] for $A=\Delta / 2$. The proof carries over to the present case since by $[8]$ the boundary Harnack principle holds for $A$ and this is what is needed.

THEOREM 2.1. The measure $P_{z}^{z_{1}}$ exists for any $z_{1} \in \partial D, z \in \bar{D}$ where $P_{z}^{z}$ is the point mass on $\omega=\omega_{z} ; \omega_{z}(0)=z, \omega_{z}(s) \in \partial$ for $s>0$.

2.5. Gauge, conditional gauge and Feynman-Kac formula. For any $q \in K_{d}$, $\int_{0}^{t} q(X(s)) d s$ is well defined where we specify $q(\partial)=0$. This follows from the theory of Markov processes as in [5] since $q \in K_{d}$ implies $G|q|$ is finite and therefore so are $G q^{+}$and $G q^{-}$and these potentials have naturally associated to them well-defined additive functionals $\int q^{+}(X(s)) d s$ and $\int q^{-}(X(s)) d s$. Thus we can set

$$
e_{q}(t)=\exp \left\{-\int_{0}^{t} q(X(s)) d s\right\} .
$$

The gauge (of $(A, D, q))$ is then defined as

$$
F(x)=E^{x}\left[e_{q}\left(\tau_{D}\right)\right], \quad x \in D .
$$

The conditional gauge is

$$
F(x, y)=E_{y}^{x}\left[e_{q}\left(\tau_{D}\right)\right], \quad(x, y) \in \bar{D} \times \bar{D} .
$$


In the definition of the conditional gauge, $\tau_{D}$ is identically 0 under $P_{y}^{x}$ when $x=y$. The significance of the conditional gauge will become apparent in our discussion of the Feynman-Kac formula. The importance of the gauge is contained in the next result. The boundedness of $F(x)$ from 0 and $\infty$ once it is not identically $\infty$ is called the Gauge Theorem and first appeared in [12] for $A=\Delta / 2$. The other parts appeared in [30] for $A=\Delta / 2$. The condition (2.5.2) is prevalent in [1]. The proof follows exactly as for $A=\Delta / 2$ and is omitted.

THEOREM 2.2. If $F(\cdot) \not \equiv \infty$ then there exist positive constants $c_{1}$ and $c_{2}$ such that $c_{1} \leq F(x) \leq c_{2}$, for all $x \in D$. The condition $F(\cdot) \not \equiv \infty$ is equivalent to either of the following (see [11], when $q$ is bounded, a partial proof is found in [2], a complete proof can be found in [13]) :

(2.5.1) There is a solution in $D$ to $L u=0$ with $\inf _{D} u>0$.

(2.5.2) $\inf \left(\left.\operatorname{spec} L\right|_{D}\right)>0$, considering $L$ with Dirichlet data.

(2.5.3) For each $f \in C(\partial D)$ with $f \geq 0$ there is a nonnegative solution to the Dirichlet problem

$$
L u(x)=0, \quad x \in D, \quad u(z)=f(z), \quad z \in \partial D .
$$

We wish to establish now that when $F(\cdot) \not \equiv \infty$, the unique (weak) solution to the Dirichlet problem in (2.5.3) is given by $u(x)=E^{x}\left[f\left(X\left(\tau_{\bar{D}}\right)\right) e_{q}\left(\tau_{D}\right)\right]$. This is a version of the Feynman-Kac formula. Note, by the Gauge Theorem that $u(x)$ is defined for all $x \in D$. We also set $v(x)=E^{x}\left[f\left(X\left(\tau_{\bar{D}}\right)\right)\right]$ and observe $v$ is a weak solution to $A v=0$ on $D, v=f$ on $\partial D$. Now, postponing temporarily a justification of Fubini, and recalling that $q(\partial)=0$,

$$
\begin{aligned}
u(x)-v(x) & =-E^{x}\left[f\left(X\left(\tau_{\bar{D}}\right)\right) \int_{0}^{\tau_{D}} q(X(s)) \exp \left\{-\int_{s}^{\tau_{D}} q(X(t)) d t\right\} d s\right] \\
& =-\int_{0}^{\infty} E^{x}\left[q(X(s)) \exp \left\{-\int_{s}^{\tau_{D}} q(X(t)) d t\right\} f\left(X\left(\tau_{D^{-}}\right)\right)\right] d s \\
& =-\int_{0}^{\infty} E^{x}\left[q(X(s)) E^{X(s)}\left[\exp \left\{-\int_{0}^{\tau_{D}} q(X(t)) d t\right\} f\left(X\left(\tau_{D^{-}}\right)\right)\right]\right] d s \\
& =-\int_{0}^{\infty} E^{x}[q(X(s)) u(X(s))] d s \\
& =-G(u q)(x) .
\end{aligned}
$$

where the interchange of integrals is justified by the boundedness of $v, f$ and $F$, the bound (2.3.1) and the assumption $q \in K_{d}$. Thus $u(x)=v(x)-G(u q)(x)$ and applying $-A$ to both sides shows $-A u=-q u$ or $L u=0$ (in the weak sense.) What remains is to consider to what extent $u$ assumes the boundary values $f$. For this one can prove Lemma A.4.8 of [1] generalized to the present situation. Namely, for $z$ a regular point of $\partial D, \lim _{x \rightarrow z} E^{x}\left|e_{q}\left(\tau_{D}\right)-1\right|=0$. Then notice that

$$
|u(x)-v(x)| \leq\|f\|_{\infty} E^{x}\left|e_{q}\left(\tau_{D}\right)-1\right| \rightarrow 0 \quad \text { as } x \rightarrow z .
$$

But $z$ is regular so $v(x) \rightarrow f(z)$ as $x \rightarrow z$ and then so must $u(x) \rightarrow f(z)$.

The connection with the conditional gauge is now easy. Recall the notation $w^{x}(d z)=P^{x}\left(X\left(\tau_{\bar{D}}\right) \in d z\right)$ for the $A$-harmonic measure on the Borel subsets of 
$\partial D, B(\partial D)$, evaluated at $x \in D$. Then

$$
\begin{aligned}
u(x) & =E^{x}\left[f\left(X\left(\tau_{\bar{D}}\right)\right) e_{q}\left(\tau_{D}\right)\right] \\
& =\int_{\partial D} E_{z}^{x}\left[f\left(X\left(\tau_{\bar{D}}\right)\right) e_{q}\left(\tau_{D}\right)\right] w^{x}(d z) \\
& =\int_{\partial D} f(z) E_{z}^{x}\left[e_{q}\left(\tau_{D}\right)\right] w^{x}(d z) \\
& =\int_{\partial D} f(z) F(x, z) w^{x}(d z) .
\end{aligned}
$$

One of our main results is that $F(\cdot, \cdot) \simeq 1$ when $F(\cdot) \not \equiv \infty$, Theorem 4.2. Remembering that $w_{L}^{x}(d z)$ denotes $L$-harmonic measure we then have $w_{L}^{x}(d z)=$ $F(x, z) w^{x}(d z)$. It is this and other relations which we exploit in $\S 5$.

3. Green function and kernel function estimates. This section contains the statement and proof of our main analytic estimate, Theorem 3.1. The estimates give upper bounds for the Green function and kernel function associated to the conditioned process for $A$ in terms of the Newtonian potential for $\Delta / 2$. We shall call inequality (3.1.1) from Theorem 3.1, the $3 \mathrm{G}$ Theorem.

THEOREM 3.1. For a bounded Lipschitz domain $D \subseteq R^{d}$ and $A \in \mathcal{E}(\lambda)$ there exists a positive constant $c$ depending on $\lambda, d$, the diameter and the Lipschitz character of $D$ such that

$$
G(x, z)^{-1} G(x, y) G(y, z) \leq c\left\{|x-y|^{2-d}+|y-z|^{2-d}\right\}, \quad x, y, z \in D,
$$

$$
K(x, z)^{-1} G(x, y) K(y, z) \leq c\left\{|x-y|^{2-d}+|y-z|^{2-d}\right\}, \quad x, y \in D, z \in \partial D .
$$

PROOF. The bound in (3.1.2) follows from (3.1.1) since the Martin boundary for $A$ on $D$ is $\partial D$ by [8] and

$$
\lim _{w \rightarrow z} G(x, w)^{-1} G(x, y) G(y, w)=K(x, z)^{-1} G(x, y) K(y, z) .
$$

The proof consists of a lemma and four cases. First, however, we define the following items:

$\delta(x)=\operatorname{dist}(x, \partial D)$

$x^{*}=$ a point on $\partial D$ such that $\delta(x)=\left|x-x^{*}\right|$, and let $A_{r}\left(x^{*}\right)$ be so that $\delta\left(A_{r}\left(x^{*}\right)\right) \geq c_{1} r$ and $\left|A_{r}\left(x^{*}\right)-x^{*}\right|=r$. The constant $c_{1}<1$ depends only on the Lipschitz character of $D$ and defining such an $A_{r}\left(x^{*}\right)$ is possible whenever $r<r_{0}$ where again $r_{0}$ depends only on the Lipschitz character of $D$. Finally, set

$$
\tilde{x}_{r}=\left\{\begin{array}{ll}
x & \text { if } \delta(x) \geq \delta\left(A_{r}\left(x^{*}\right)\right), \\
\tilde{x}_{r}=A_{r}\left(x^{*}\right) & \text { if } \delta(x)<\delta\left(A_{r}\left(x^{*}\right)\right),
\end{array} \quad \text { for } 0<r<r_{0},\right.
$$

LEMMA 3.2. For $0<r<r_{0}$ suppose $|x-y|>3 r$ and $|x-z|>3 r$. If (3.1.1) holds for $\left(\tilde{x}_{r}, y, z\right)$ then it holds for $(x, y, z)$.

PROOF. When $\delta(x) \geq \delta\left(A_{r}\left(x^{*}\right)\right), \tilde{x}_{r}=x$ and there is nothing to prove so assume $\delta(x)<\delta\left(A_{r}\left(x^{*}\right)\right)$. When $\delta(x)<\delta\left(A_{r}\left(x^{*}\right)\right)$ then $\delta\left(\tilde{x}_{r}\right) \simeq r$ and by the boundary Harnack principle, $G\left(\tilde{x}_{r}, z\right)^{-1} G\left(\tilde{x}_{r}, y\right) \simeq G(x, z)^{-1} G(x, y)$. However,

$$
\left|\tilde{x}_{r}-y\right| \geq|x-y|-\left|\tilde{x}_{r}-x\right| \geq|x-y|-r \geq \frac{2}{3}|x-y| \text {. }
$$


This implies (3.1.1) holds for $(x, y, z)$.

In all the cases presented below we assume $\delta(x) \leq \delta(z)$ by reason of symmetry. We shall fix a constant $c_{2}<1 / 9$ so that $c_{2}|x-z|<r_{0}$ for all $x, z \in D$. This is possible since $D$ is assumed bounded. Set $c_{3}=\left(c_{2}^{-1}+2\right)$.

Case 1. $|x-z| \leq c_{3} c_{1}^{-1} \delta(x)$.

If $|x-z| \leq \delta(x) / 2$ then by (2.3.2) we would have $G(x, z) \geq c|x-z|^{2-d}$, and by $(2.3 .1)$

$$
G(x, z)^{-1} G(x, y) G(y, z) \leq c|x-z|^{d-2}|x-y|^{2-d}|y-z|^{2-d}
$$

which implies (3.1.1).

On the other hand, when $|x-z|>\delta(x) / 2$ select a point $\tilde{z}$ with $|x-\tilde{z}|=$ $\delta(x) / 2$. Then by Harnack's principle $G(x, z) \simeq G(x, \tilde{z})$ and by $(2.3 .2)$ the inequality $G(x, \tilde{z}) \geq c|x-\tilde{z}|^{2-d}$ holds. Now $\delta(x) / 2<|x-z| \leq c \delta(x)$ and $|x-\tilde{z}|=\delta(x) / 2$. Thus $|x-\tilde{z}| \simeq|x-z|$ and (3.1.1) holds.

Case 2. $|x-y| \leq c_{1}^{-1}\left(c_{2}^{-1}+1\right) \delta(x),|x-z|>c_{3} c_{1}^{-1} \delta(x)$.

In this case, $\left|z-x^{*}\right| \geq\left(c_{3} c_{1}^{-1}-1\right) \delta(x)$ and $\left|y-x^{*}\right| \leq\left(c_{1}^{-1}\left(c_{2}^{-1}+1\right)+1\right) \delta(x)$. Now when $\delta(y)<\delta(x)$ use Carleson's estimate to conclude $G(y, z) \leq c G\left(A_{\delta(x)}\left(y^{*}\right), z\right)$. However, there is a Harnack chain in $D \backslash\{z\}$ from $A_{\delta(x)}\left(y^{*}\right)$ to $x$ of length independent of $\delta(x)$ so that $G(x, z) \simeq G\left(A_{\delta(x)}\left(y^{*}\right), z\right)$. Thus $G(y, z) \leq c G(x, z)$ and with (2.3.1) applied to $G(x, y)$, this implies (3.1.1). When $\delta(y) \geq \delta(x)$ then by the Harnack chain argument used above $G(y, z) \simeq G(x, z)$ and together with (2.3.1) applied to $G(x, y)$ we again have (3.1.1).

Case 3. $|x-z|>c_{3} c_{1}^{-1} \delta(x),|y-z| \geq 2|x-z|,|x-y|>c_{1}^{-1}\left(c_{2}^{-1}+1\right) \delta(x)$.

Set $r=c_{2}|x-z|$ so that $r<r_{0}$. Then $|y-z| \geq 3 r,|x-z| \geq 3 r$ and by Lemma 3.2 it suffices to consider $\left(x, y, \tilde{z}_{r}\right)$. Observing that

$$
|x-y| \geq|y-z|-|x-z| \geq|x-z| \geq 3 r
$$

and

$$
\left|x-\tilde{z}_{r}\right| \geq|x-z|-r \geq(9-1) r \geq 3 r
$$

we see that by another application of Lemma 3.2 it suffices to consider $\left(\tilde{x}_{r}, y, \tilde{z}_{r}\right)$. But

$$
\left|\tilde{x}_{r}-\tilde{z}_{r}\right| \leq|x-z|+2 r \leq\left(c_{2}^{-1}+2\right) r \leq c_{3} c_{2}^{-1}\left(\delta\left(\tilde{x}_{r}\right) \wedge \delta\left(\tilde{z}_{r}\right)\right),
$$

i.e. we are back in Case 1 and (3.1.1) holds.

Case 4. $|x-z|>c_{3} c_{1}^{-1} \delta(x),|x-y|>c_{1}^{-1}\left(c_{2}^{-1}+1\right) \delta(x),|y-z|<2|x-z|$.

Set $r=c_{2}|x-y|$. Then observing $|x-y|=c_{2}^{-1} r$ and

$$
|x-z| \geq|x-y|-|y-z| \geq|x-y|-2|x-z|
$$

which implies $|x-z| \geq \frac{1}{3}|x-y| \geq 3 r$ we see that Lemma 3.2 applies. Having switched to $\left(\tilde{x}_{r}, y, z\right)$ we note that

$$
\left|\tilde{x}_{r}-y\right| \leq|x-y|+r=c_{2}^{-1} r+r \leq c_{1}^{-1}\left(c_{2}^{-1}+1\right) \delta\left(x_{r}\right) .
$$

If $\delta\left(\tilde{x}_{r}\right) \leq \delta(z)$ we are in Case 1 or 2 and are done. If $\delta\left(\tilde{x}_{r}\right)>\delta(z)$ we would be through should $\left(z, y, \tilde{x}_{r}\right)$ satisfy the conditions of either of the first three cases. So we may assume the worst, that $\left(z, y, \tilde{x}_{r}\right)$ falls into Case 4 . However, in that event, set $s=c_{2}|y-z|$. Then with the same argument we used in the first part of Case 4 it follows that either $\left(\tilde{z}_{s}, y, \tilde{x}_{r}\right)$ or $\left(\tilde{x}_{r}, y, \tilde{z}_{s}\right)$ satisfies Case 1 or 2 and that completes the proof of Theorem 3.1.

In the course of proving the continuity of $E_{y}^{x}\left[e_{q}\left(\tau_{D}\right)\right]$ on $\bar{D} \times \bar{D}$ we shall need the following estimate. We are of course still assuming $D$ is Lipschitz and $A \in \mathcal{E}(\lambda)$. 
ThEOREM 3.3. For any $z \in \bar{D}, w \in D, w \neq z$,

$$
\lim _{x, y \rightarrow z} G(x, y)^{-1} G(x, w) G(w, y)=0 .
$$

Also, if $z, z^{\prime} \in \partial D$, then

$$
\lim _{\substack{x \rightarrow z \\ y \rightarrow z^{\prime}}} G(x, y)^{-1} G(x, w) G(w, y)=K\left(z, w, z^{\prime}\right)
$$

exists and is a continuous function of $\left(z, z^{\prime}\right)$ on $\partial D \times \partial D$.

ProOF. For $z \in D$, by the continuity of solutions to $A u=0, G(x, w) \rightarrow G(z, w)$ and $G(w, y) \rightarrow G(w, z)$. As for the denominator, $G(x, y) \geq c|x-y|^{2-d}$ for $x$ and $y$ sufficiently close to $z$. Thus the limit in (3.3.1) is 0 .

When $z \in \partial D$, let $|x-y|=3 r$. Define $x^{*}$ and $y^{*}$ to be the points on $\partial D$ closest to $x$ and $y$. Also take $x_{r}$ and $y_{r}$ to be points in $D$ so that $\left|x^{*}-x_{r}\right|=r,\left|y^{*}-y_{r}\right|=r$ for $r$ small, $\delta\left(x_{r}\right) \simeq r$ and $\delta\left(y_{r}\right) \simeq r, 5 r \geq\left|y_{r}-x_{r}\right| \geq r$. Applying the boundary Harnack principle twice, we obtain

$$
\begin{aligned}
G(x, y)^{-1} G(x, w) G(w, y) & \leq c G\left(x_{r}, y\right)^{-1} G\left(x_{r}, w\right) G(w, y) \\
& \leq c G\left(x_{r}, y_{r}\right)^{-1} G\left(x_{r}, w\right) G\left(w, y_{r}\right)
\end{aligned}
$$

As a consequence of the above inequalities for $x_{r}$ and $y_{r}$ it follows that $G\left(x_{r}, y_{r}\right) \geq$ $c\left|x_{r}-y_{r}\right|^{2-d} \geq c r^{2-d}$. Thus

$$
\lim _{x, y \rightarrow z} G(x, y)^{-1} G(x, w) G(w, y) \leq c \lim _{r \rightarrow 0} r^{d-2} G\left(x_{r}, w\right) G\left(w, y_{r}\right)=0 .
$$

For the second claim (3.3.2), let $z \neq z^{\prime}$ and select a compact set $K$ with $z \in K$, $z^{\prime} \notin K$. Then we claim that

$$
G(x, y)^{-1} G(x, w) G(w, y) \rightarrow K\left(x, z^{\prime}\right)^{-1} G(x, w) K\left(w, z^{\prime}\right) \quad \text { uniformly on } K \cap \bar{D} \text {. }
$$

The convergence is uniform on a subcompact $K(r)=\{x \in K \cap D: \delta(x) \geq r\}$. To fill in up to the boundary note that $G(x, y)^{-1} G(x, w) G(w, y)$ extends to a Hölder continuous function on $K \cap \bar{D}$ with the Hölder exponent independent of $y$, as $y \rightarrow z^{\prime}$. Thus, this forms an equicontinuous bounded family of continuous functions, in $x$. Since the sequence converges pointwise on $K \cap \bar{D}$ it converges uniformly there. This uniform convergence justifies the exchange of limits

$$
\lim _{\substack{x \rightarrow z \\ y \rightarrow z^{\prime}}} G(x, y)^{-1} G(x, w) G(w, y)=\lim _{x \rightarrow z} K\left(x, z^{\prime}\right)^{-1} G(x, w) K\left(w, z^{\prime}\right)=K\left(z, w, z^{\prime}\right)
$$

and again this last limit exists and is Hölder continuous in $z$ by the boundary Harnack principle. If $z^{\prime}, z \rightarrow z^{\prime \prime} \in \partial D$ we need to show $K\left(z, w, z^{\prime}\right) \rightarrow 0$. This, however, mimics the first part, if $\left|z-z^{\prime}\right|=3 r$ select $x_{r}$ and $y_{r}$ with $\left|x_{r}-z\right|<r$, $\delta\left(x_{r}\right)>r / 2,\left|y_{r}-z^{\prime}\right|<r, \delta\left(y_{r}\right)>r / 2$. Then $\left|x_{r}-y_{r}\right|>r$. Then given $\varepsilon>0$

$$
\left|G\left(x_{r}, y_{r}\right)^{-1} G\left(x_{r}, w\right) G\left(w, y_{r}\right)-K\left(z, w, z^{\prime}\right)\right|<\varepsilon
$$

provided $r$ is sufficiently small. Now $x_{r}, y_{r} \rightarrow z^{\prime \prime}$ so by the first part

$$
G\left(x_{r}, y_{r}\right)^{-1} G\left(x_{r}, w\right) G\left(w, y_{r}\right) \rightarrow 0 \text {. }
$$

Thus $K\left(z, w, z^{\prime}\right) \rightarrow 0$ as $z, z^{\prime} \rightarrow z^{\prime \prime} \in \partial D$.

In the course of the proof of the Conditional Gauge Theorem we will need to know $P_{y}^{x}\left(\tau_{D}<\infty\right)=1$. More can be said, in fact $E_{y}^{x} \tau_{D}<\infty$. For the case $A=\Delta / 2$ this was shown in [14]. [4] has a similar result for $A \in \mathcal{E}(\lambda)$, when $\partial D$ is Lipschitz. 
THEOREM 3.4. There exists a positive constant depending on $\lambda$ and the Lipschitz character of $D$ and the diameter of $D$ such that

$$
E_{y}^{x} \tau_{D} \leq c(m(D))^{2 / d}, \quad x, y \in \bar{D}
$$

ProOF. For $x, y \in D$,

$$
\begin{aligned}
E_{y}^{x} \tau_{D} & =G(x, y)^{-1} \int_{D} G(x, w) G(w, y) d w \\
& \leq c \int_{D}\left\{|x-w|^{2-d}+|w-y|^{2-d}\right\} d w, \quad \text { by Theorem } 3.1 \\
& \leq c(m(D))^{2 / d} .
\end{aligned}
$$

When $x \in D, y \in \partial D$,

$$
E_{y}^{x} \tau_{D}=K(x, y)^{-1} \int_{D} G(x, w) K(w, y) d w
$$

and use Theorem 3.1 again to obtain $E_{y}^{x} \tau_{D} \leq c(m(D))^{2 / d}$. When $x, y \in \partial D$, if $x=y E_{x}^{x} \tau_{D}=0$. If $x \neq y$ let $x_{n} \in D$ converge to $x \in \partial D$. Then

$$
E_{y}^{x_{n}} \tau_{D}=K\left(x_{n}, y\right)^{-1} \int_{D} G\left(x_{n}, w\right) K(w, y) d w .
$$

By boundary Harnack and weak maximum principles $\lim _{x_{n} \rightarrow x} G\left(x_{n}, w\right) / K\left(x_{n}, y\right)$ exists for each $w \in D$ and Theorem 3.1 allows us to use dominated convergence to conclude $E_{y}^{x} \tau_{D} \leq c(m(D))^{2 / d}$. The last case $x \in \partial D, y \in D$ is similar, with $x_{n}$ as above,

$$
E_{y}^{x_{n}} \tau_{D}=G\left(x_{n}, y\right)^{-1} \int_{D} G\left(x_{n}, w\right) G(w, y) d w \leq c(m(D))^{2 / d}
$$

Then $G\left(x_{n}, w\right) / G\left(x_{n}, y\right) \rightarrow K(w, x) / K(y, x)$ and we have

$$
E_{y}^{x_{n}} \tau_{D} \rightarrow E_{y}^{x} \tau_{D}=K(y, x)^{-1} \int_{D} G(y, w) K(w, x) d w \leq c(m(D))^{2 / d}
$$

by Theorem 3.1 .

4. The Conditional Gauge Theorem. In [10], Chung has presented a nice simplification of the arguments of [28]. This simplification, with Theorems 3.1 and 3.4 , give immediately

THEOREM 4.1. If $F\left(x_{0}, z_{0}\right)<\infty$ for some $x_{0} \in D, z_{0} \in \partial D$ then there exist positive constants $c_{1}$ and $c_{2}$ with

$$
c_{1} \leq F(x, z) \leq c_{2} \quad \text { for all } x \in D, z \in \partial D
$$

We now state a Conditional Gauge Theorem with weaker hypotheses and strong er conclusion than Theorem 4.1. Namely, the conditional gauge is assumed to be finite somewhere on $\bar{D} \times \bar{D} \backslash \operatorname{diag}(\bar{D} \times \bar{D})$ and shown to be bounded above and below on $\bar{D} \times \bar{D}$ as a consequence.

THEOREM 4.2. If there exists a pair $\left(x_{0}, y_{0}\right) \in \bar{D} \times \bar{D}$ with $x_{0} \neq y_{0}$ and $F\left(x_{0}, y_{0}\right)=E_{y_{0}}^{x_{0}}\left[e_{q}\left(\tau_{D}\right)\right]<\infty$ then

$$
0<\inf _{\substack{x \in \bar{D} \\ y \in \bar{D}}} F(x, y) \leq \sup _{\substack{x \in \bar{D} \\ y \in \bar{D}}} F(x, y)<\infty .
$$

The proof of this result will be broken into several parts. 
LEMMA 4.3. There is a $\delta>0$ such that for any Borel set $A \subseteq D$ with $m(A)<\delta$ and any $S \subseteq \Omega$ and $\Lambda \subseteq \bar{D} \times \bar{D}$ such that $P_{y}^{x}(S) \geq c>0$ for $(x, y) \in \Lambda$, we have $E_{y}^{x}\left[S ; e_{q}\left(\tau_{A}\right)\right] \simeq 1$ on $\Lambda$.

PROOF. Since

$$
\begin{aligned}
& E_{y}^{x}\left[\int_{0}^{\tau_{A}}|q|(X(s)) d s\right] \leq E_{y}^{x}\left[\int_{0}^{\tau_{D}} 1_{A}|q|(X(s)) d s\right] \\
& \quad=\int_{A} G(x, y)^{-1} G(x, w) G(w, y)|q|(w) d w, \quad \text { when } y \in D
\end{aligned}
$$

or $\int K(x, y)^{-1} G(x, w) K(w, y)|q|(w) d w$ when $y \in \partial D$. When $x \in \partial D$ and $y \in D$ the upper bound is $\int_{A} K(y, x)^{-1} K(w, x) G(w, y)|q|(w) d w$ and for $x, y \in \partial D$ it becomes $\int_{A} K(x, w, y)|q|(w) d w$ (see Theorem 3.3) and in all cases Theorem 3.1 implies there exists a $\delta>0$ such that if $m(A)<\delta$ then

$$
E_{y}^{x}\left[\int_{0}^{\tau_{A}}|q|(X(s)) d s\right]<\frac{1}{2}, \quad(x, y) \in \bar{D} \times \bar{D} .
$$

Thus by Khasminski's lemma [1]

$$
E_{y}^{x}\left[e_{q}\left(\tau_{A}\right) ; S\right] \leq E_{y}^{x}\left[e_{|q|}\left(\tau_{A}\right)\right] \leq 1 /(1-1 / 2)=2,
$$

and the upper bound holds. For the lower bound use Jensen's inequality, for $(x, y)$ $\in \Lambda$

$$
\begin{aligned}
E_{y}^{x}\left[e_{q}\left(\tau_{A}\right) ; S\right] & =P_{y}^{x}[S] E_{y}^{x}\left[e_{q}\left(\tau_{A}\right) \mid S\right] \\
& \geq c \exp \left\{E_{y}^{x}\left[\int_{0}^{\tau_{A}} q(X(s)) d s \mid S\right]\right\} \\
& \geq c \exp \{-1 / 2\} .
\end{aligned}
$$

For $a>0$ define the set $D(a)=:\{x \in D: d(x, \partial D)<a\}$. Now $D(a)$ need not be connected (if $D$ is an annulus for example) so select an open connected set $C(a) \supseteq D(a)$. In fact given the $\delta$ in Lemma 4.3 we select $a$ and $C(a)$ so that $m(C(a))<\delta$. We also will select $C(a / 2) \supseteq \overline{C(a / 4)}, C(a / 4) \supset \overline{C(a / 8)}$ in such a way that $C\left(a 2^{-k}\right) \supseteq D\left(a 2^{-k}\right)$, for $k=1,2,3$, each is connected, and

$$
\operatorname{dist}\left(C\left(a 2^{-j}\right)^{c}, C\left(a 2^{-k}\right)\right) \geq \eta a \text { for some small } \eta>0,
$$

whenever $j<k$. Define for $k=0,1,2,3$

$$
U\left(a 2^{-k}\right)=D \backslash \overline{C\left(a 2^{-k}\right)} \quad \text { and } \quad l\left(a 2^{-k}\right)=\overline{U\left(a 2^{-k}\right)} \cap \overline{C\left(a 2^{-k}\right)} .
$$

LEMMA 4.4. There exists a positive constant $c$ such that

$$
P_{y}^{x}\left(\tau_{C(a)}=\tau_{D}\right) \geq c, \quad x \in l(a / 2), y \in \overline{C(a / 4)} .
$$

Furthermore, there is a $\beta>0$ such that if $0<r<a / 8$, then

$$
P_{y}^{x}\left(\tau_{B(y, r)}=\tau_{D}\right) \geq c, \quad y \in \overline{U(a / 8)}, x \in \partial B(y, \beta r) .
$$

ProOF. Set $l(a / 2 ; \eta a / 2)=\{x: \operatorname{dist}(x, l(a / 2)) \leq \eta a / 2\}$, and let $f(x, y) \equiv$ $P_{y}^{x}\left(\tau_{C(a)}<\tau_{D}\right)$. We claim $f(x, y)$ is a continuous function on $l(a / 2 ; \eta a / 2) \times C(a / 4)$. To see this, let $K_{a}(x, w)$ denote the kernel function for $A$ on $C(a), w \in l(a)$, 
normalized at some $z_{0} \in C(a)$ so that $K_{a}\left(z_{0}, w\right) \equiv 1$. Also, let $\omega_{a}^{z_{0}}$ be harmonic measure for $C(a)$ evaluated at $z_{0}$. Then,

$$
\begin{aligned}
f(x, y) & =G(x, y)^{-1} E^{x}\left[G\left(X\left(\tau_{C(a)}\right), y\right) ; \tau_{C(a)}<\tau_{D}\right] \\
& =G(x, y)^{-1} \int_{l(a)} K_{a}(x, w) G(w, y) \omega_{a}^{z_{0}}(d w) .
\end{aligned}
$$

Now $K_{a}(x, w) \leq c G(x, w)$ for $(x, w) \in l(a / 2 ; \eta a / 2) \times l(a)$ so by the $3 \mathrm{G}$ Theorem, $G(x, y)^{-1} K_{a}(x, w) G(w, y)$ is bounded for $(x, w, y) \in l(a / 2 ; \eta a / 2) \times l(a) \times \overline{C(a / 4)}$. It is continuous in $(x, y) \in l(a / 2 ; \eta a / 2) \times \overline{C(a / 4)}$ for each $w \in l(a)$. The continuity in $y$ up to $\partial D$ follows from the boundary Harnack and maximum principles which give convergence of $G(x, y)^{-1} G(w, y)$ uniformly in $(x, w) \in l(a / 2 ; \eta a / 2) \times l(a)$ as $y \rightarrow z \in$ $\partial D$. Thus by bounded convergence, $f(x, y)$ is continuous on $l(a / 2 ; \eta a / 2) \times \overline{C(a / 4)}$. Next we claim $f(x, y)<1-c$ for some positive $c$ and all $(x, y) \in l(a / 2) \times \overline{C(a / 4)}$. Otherwise suppose $f(x, y)=1$ for some $(x, y) \in l(a / 2) \times \overline{C(a / 4)}$. Then selecting a positive $r$ with $r<\eta a / 2$ and setting $\tau_{r}=\inf \{t>0:|X(t)-X(0)|>r\}, f(x, y)=$ $E_{y}^{x} f\left(X\left(\tau_{r}\right), y\right)$. Thus, since $f(u, v) \leq 1$ it follows that $f(u, y)=1$ for almost every $u$ with respect to $P_{y}^{x}\left(X\left(\tau_{r}\right) \in d u\right)$. This measure is absolutely continuous with respect to $P^{x}\left(X\left(\tau_{r}\right) \in d u\right)$. Though this last measure may not be absolutely continuous with respect to surface measure on $\partial B(x, r)$ it does satisfy the doubling condition: there is a $c>0$ such that $\forall u \in \partial B(x, r)$,

$$
P^{x}\left(X\left(\tau_{r}\right) \in B(u, s) \cap \partial B(x, r)\right) \geq c P^{x}\left(X\left(\tau_{r}\right) \in B(u, 2 s) \cap \partial B(x, r)\right),
$$

see [8]. Thus $P^{x}\left(X\left(\tau_{r}\right) \in d u\right)$ gives positive mass to any open set on $\partial B(x, r)$ and therefore $f(u, y)=1$ on a dense set of points $u$ on $\partial B(x, r)$ and by continuity, $f(u, y) \equiv 1$ on $\partial B(x, r)$. Thus $f(u, y) \equiv 1$ on $B(x, r)$ and by connectedness of $l(a / 2)$ it follows that $f(u, y) \equiv 1$ for $u$ in $l(a / 2)$. This, of course, is a contradiction and the claim is proved, and consequently (4.4.1) holds.

For (4.4.2) take $r<a / 8$ and $\beta$ small. Then when $x \in \partial B(y, \beta r)$ and $y \in \overline{U(a / 8)}$ we have

$$
|x-y|=\beta r<\beta \frac{a}{8} \quad \text { and } \quad \delta(x) \geq \delta(y)-|x-y| \geq \frac{a}{8}-\beta \frac{a}{8}=(1-\beta) \frac{a}{8}
$$

so that $|x-y| \leq[\beta /(1-\beta)] \delta(x)$. Thus we can apply (2.3.1) and (2.3.2) to obtain

$$
g(x, y) \equiv P_{y}^{x}\left(\tau_{B(y, r)}<\tau_{D}\right)=G(x, y)^{-1} E^{x}\left[G\left(X\left(\tau_{B(y, r)}\right), y\right)\right] \leq c(\beta)<1,
$$

by selecting $\beta$ sufficiently small. Thus, fixing such a $\beta$, for some positive $c$, $P_{y}^{x}\left(\tau_{B(y, r)}=\tau_{D}\right) \geq c$ for $x \in \partial B(y, \beta r), y \in \overline{U(a / 8)}$.

Define the following sequences of stopping times:

(a) For $y \in \overline{U(a / 8)}$ and $r<a / 8$, set $S_{0}=0$, and $\beta$ as in Lemma 4.4:

$$
\begin{aligned}
S_{2 n-1} & =S_{2 n-2}+T_{B(y, \beta r)} \circ \theta_{S_{2 n-2}}, \\
S_{2 n} & =S_{2 n-1}+\tau_{B(y, r)} \circ \theta_{S_{2 n-1}}, \quad n=1,2, \ldots,
\end{aligned}
$$

where $T_{B}$ is the hitting time at $B, T_{B}=\inf \left\{t>0: X_{t} \in B\right\}$. The times $S_{k}$ depend on $y$ and $r$ and when confusion may arise we shall write $S_{k}(y, r)$.

(b) For $y \in \overline{C(a / 4)}$, set $T_{0}=0$,

$$
\begin{aligned}
T_{2 n-1} & =T_{2 n-2}+\tau_{U(a / 2)} \circ \theta_{T_{2 n-2},} \\
T_{2 n} & =T_{2 n-1}+\tau_{C(a)} \circ \theta_{T_{2 n-1}}, \quad n=1,2, \ldots
\end{aligned}
$$


We also define the sets

$$
\begin{aligned}
& A_{1}=\{(x, y) \in \overline{U(a / 2)} \times \overline{U(a / 8)}:|x-y| \geq r / 2\}, \\
& A_{2}=\overline{U(a / 2)} \times \overline{C(a / 4)},
\end{aligned}
$$

and the functions

$$
\begin{aligned}
& F_{1}\left(x, y^{*}, y\right)=\sum_{n=1}^{\infty} E_{y^{*}}^{x}\left[e_{q}\left(S_{2 n-1}(y, r)\right) ; S_{2 n-2}(y, r)<\tau_{D}\right], \\
& \text { for }(x, y) \in A_{1}, y^{*} \in B(y, \beta r / 2), \\
& F_{2}(x, y)=\sum_{n=1}^{\infty} E_{y}^{x}\left[e_{q}\left(T_{2 n-1}\right) ; T_{2 n-2}<\tau_{D}\right], \quad \text { for }(x, y) \in A_{2} .
\end{aligned}
$$

Assume that $a$ is sufficiently small so that both $x_{0} \in \overline{U(a / 2)}$ and $m(C(a))<\delta$ with $\delta$ as in Lemma 4.3. Then select $r$ so that $r<a / 8$ and $\left|x_{0}-y_{0}\right| \geq \beta r$. We will then show there exist constants $c_{1}$ and $c_{2}$, depending on $a$ and $r$ such that $c_{1} \leq F(x, y) \leq c_{2}$. But then we can find a new pair $\left(x_{0}^{\prime}, y_{0}^{\prime}\right), x_{0}^{\prime} \neq y_{0}^{\prime}$, with $F\left(x_{0}^{\prime}, y_{0}^{\prime}\right)<\infty$ and $x_{0}^{\prime} \in U\left(a^{\prime} / 8\right),\left|x_{0}-y_{0}\right| \geq r^{\prime} / 2$ with $a^{\prime}, r^{\prime}$ depending on $D$ alone. Then we can retrace our steps to prove there exist constants $c_{1}$ and $c_{2}$ independent of the original $a$ and $r$ such that $c_{1} \leq F(x, y) \leq c_{2}$. The case $x_{0} \in \partial D$ is treated later.

LEMMA 4.5. The following equivalences hold:

$$
\begin{aligned}
& F\left(x, y^{*}\right) \simeq F_{1}\left(x, y^{*} ; y\right), \quad \text { for }(x, y) \in A_{1}, y^{*} \in B(y, \beta r / 2), \\
& F \simeq F_{2} \text { on } A_{2}, \\
& F_{1}\left(x, y^{*} ; y\right) \simeq F_{1}(x, y ; y), \quad \text { for }(x, y) \in A_{1}, y^{*} \in B(y, \beta r / 2), \\
& F_{2}(x, y) \simeq F_{2}\left(x, y^{*}\right) \quad \text { for }(x, y),\left(x, y^{*}\right) \in A_{2} .
\end{aligned}
$$

PROOF. For $(x, y) \in A_{1}, y^{*} \in B(y, \beta r / 2)$ writing $S_{k}$ for $S_{k}(y, r)$, by Theorem 3.3 ,

$$
\begin{aligned}
F(x, y) & =\sum_{n=1}^{\infty} E_{y}^{x}\left[e_{q}\left(\tau_{D}\right) ; S_{2 n}=\tau_{D}\right] \\
& =\sum_{n=1}^{\infty} E_{y}^{x}\left[e_{q}\left(S_{2 n-1}\right) E_{y}^{X\left(S_{2 n-1}\right)}\left[e_{q}\left(\tau_{D}\right) ; \tau_{D}=\tau_{B(y, r)}\right] ; S_{2 n-2}<\tau_{D}\right] \\
& \simeq \sum_{n=1}^{\infty} E_{y^{*}}^{x}\left[e_{q}\left(S_{2 n-1}\right) ;\left(S_{2 n-1}\right) ; S_{2 n-2}<\tau_{D}\right], \quad \text { by }(4.4 .2) \text { and Lemma } 4.3 \\
& =F_{1}\left(x, y^{*} ; y\right)
\end{aligned}
$$

and (4.5.1) is proved.

Similarly, for $(x, y) \in A_{2}$, Theorem 3.3 implies $E_{y}^{x} \tau_{D}<\infty$ and

$$
\begin{aligned}
F(x, y) & =\sum_{n=1}^{\infty} E_{y}^{x}\left[e_{q}\left(\tau_{D}\right) ; \tau_{D}=T_{2 n}\right] \\
& =\sum_{n=1}^{\infty} E_{y}^{x}\left[e_{q}\left(T_{2 n-1}\right) E_{y}^{X\left(T_{2 n-1}\right)}\left[e_{q}\left(\tau_{D}\right) ; \tau_{D}=\tau_{C(a)}\right] ; T_{2 n-2}<\tau_{D}\right]
\end{aligned}
$$

and Lemma 4.3 and (4.4.1) imply part (4.5.2) holds. 
Let now $(x, y) \in A_{1}, y^{*} \in B(y, \beta r / 2), u, u^{*} \in \partial B(y, \beta r)$. Notice that since $r<a / 8$,

$$
\operatorname{dist}(u, \partial D)>a / 16, \quad \operatorname{dist}\left(u^{*}, \partial D\right)>a / 16 .
$$

We claim that since $y \in B(x, \beta r)$

$$
c^{-1} \frac{G\left(u^{*}, y^{*}\right)}{G\left(x, y^{*}\right)} \leq \frac{G(u, y)}{G(x, y)} \leq c \frac{G\left(u^{*}, y^{*}\right)}{G\left(x, y^{*}\right)},
$$

where $c$ may depend on $a$ and $r$. Using (2.3.1) and (2.3.2), we see $G\left(u^{*}, y^{*}\right) \simeq r^{2-d}$ and $G(u, y) \simeq r^{2-d}$ (in fact, the constant in these equivalences is independent of $a$ and $r)$. Thus it remains to establish that $c^{-1} G(x, y) \leq G\left(x, y^{*}\right) \leq c G(x, y)$. But this is just Harnack's inequality applied, in $B(y, 3 \beta r / 4)$ say, to $G(x, \cdot)$. Using this we have (writing $S_{k}$ for $S_{k}(y, r)$ )

$$
\begin{aligned}
F_{1}(x, y ; y) & =\sum_{n=1}^{\infty} E_{y}^{x}\left[e_{q}\left(S_{2 n-1}\right) ; S_{2 n-2}<\tau_{D}\right] \\
& =\sum_{n=1}^{\infty} G(x, y)^{-1} E^{x}\left[G\left(X\left(S_{2 n-1}\right), y\right) e_{q}\left(S_{2 n-1}\right) ; S_{2 n-2}<\tau_{D}\right] \\
& \simeq \sum_{n=1}^{\infty} G(x, y)^{-1} G(u, y) E^{x}\left[e_{q}\left(S_{2 n-1}\right) ; S_{2 n-2}<\tau_{D}\right] \\
& \simeq \sum_{n=1}^{\infty} G\left(x, y^{*}\right)^{-1} E^{x}\left[G\left(X\left(S_{2 n-1}\right), y^{*}\right) e_{q}\left(S_{2 n-1}\right) ; S_{2 n-2}<\tau_{D}\right] \\
& =\sum_{n=1}^{\infty} E_{y^{*}}^{x}\left[e_{q}\left(S_{2 n-1}\right) ; S_{2 n-2}<\tau_{D}\right] \\
& =F_{1}\left(x, y^{*} ; y\right),
\end{aligned}
$$

and (4.5.3) holds.

Finally, let $(x, y),\left(x, y^{*}\right) \in A_{2}$. Again by the boundary Harnack principle there exist positive constants $c_{1}$ and $c_{2}$ depending on $a$ such that

$$
c_{1} \leq G(u, y) / G(x, y) \leq c_{2} \quad \text { for } x, u \in \overline{U(a / 2)}, y \in \overline{C(a / 4)}
$$

Thus, since $X\left(T_{2 n-1}\right) \in \overline{U(a / 2)}$ on $\left\{T_{2 n-2}<\tau_{D}\right\}$,

$$
\begin{aligned}
F_{2}(x, y) & =\sum_{n=1}^{\infty} G(x, y)^{-1} E^{x}\left[e_{q}\left(T_{2 n-1}\right) G\left(X\left(T_{2 n-1}\right), y\right) ; T_{2 n-2}<\tau_{D}\right] \\
& \simeq \sum_{n=1}^{\infty} G\left(x, y^{*}\right)^{-1} E^{x}\left[e_{q}\left(T_{2 n-1}\right) G\left(X\left(T_{2 n-1}\right), y^{*}\right) ; T_{2 n-2}<\tau_{D}\right] \\
& =F_{2}\left(x, y^{*}\right),
\end{aligned}
$$

as desired.

ProOF (OF ThEOREM 4.2). For $y \in \bar{D}$ with $\left|y-x_{0}\right| \geq r / 2$ we claim

$$
F\left(x_{0}, y\right) \simeq 1 \text {. }
$$


The first case is $y_{0} \in \overline{C(a / 8)}$; then using $y_{0}$ as the $y^{*}$ in (4.5.4) and also using (4.5.2) we have for $y \in \overline{C(a / 4)}, F\left(x_{0}, y_{0}\right) \simeq F_{2}\left(\ddot{x}_{0}, y_{0}\right) \simeq F_{2}\left(x_{0}, y\right) \simeq F\left(x_{0}, y\right)$. When $y \in \overline{U(a / 4)}$ and $\left|y-x_{0}\right| \geq \beta r$ let $y^{\prime}$ be the closest point to $y$ in $\overline{C(a / 4)}$. Now construct a chain of balls, $B_{1}, \ldots, B_{N}$, from $y$ to $y^{\prime}$ with radii $\beta r / 2$ such that $y$ is the center of $B_{1}, y^{\prime}$ the center of $B_{N}, B_{i} \cap B_{i+1} \neq \varnothing$ for $i=1, \ldots, N$ and each $B_{i} \subset \overline{U(a / 8)}$. We can also do this so that the center of each $B_{i}$ is at least $\beta r$ from $x_{0}$ and so that $N$ depends on $D, a$ and $\beta r$ alone. Then denoting the center of $B_{i}$ by $y_{i}$ we have by selecting a point $y_{i}^{\prime} \in B_{i} \cap B_{i+1}$

$$
F\left(x_{0}, y_{i}\right) \simeq F_{1}\left(x_{0}, y_{i}^{\prime} ; y_{i}\right) \simeq F\left(x_{0}, y_{i}^{\prime}\right)
$$

and

$$
F\left(x_{0}, y_{i+1}\right) \simeq F_{1}\left(x_{0}, y_{i}^{\prime} ; y_{i+1}\right) \simeq F\left(x_{0}, y_{i}^{\prime}\right) .
$$

Applying these equivalences repeatedly it follows that $F\left(x_{0}, y^{\prime}\right) \simeq F\left(x_{0}, y\right) \simeq 1$.

The second case to consider is when $y_{0} \in U(a / 8)$. Then again let $y^{\prime}$ be the closest point to $y_{0}$ lying in $\overline{C(a / 8)}$. Constructing a chain of balls as in the previous paragraph one has $F\left(x_{0}, y_{0}\right) \simeq F\left(x_{0}, y^{\prime}\right)$ and thus again by the first argument $F\left(x_{0}, y\right) \simeq 1$ for $y \simeq \overline{C(a / 8)}$. Then for $y \in U(a / 8)$ with $\left|y-x_{0}\right| \geq \beta r$ we can extend the equivalence $F\left(x_{0}, y\right) \simeq 1$ exactly as in the first case.

We now claim

$$
F(x, y) \simeq 1 \text { for }(x, y) \in A_{2} .
$$

By (1) $F\left(x_{0}, z\right) \simeq 1$ for $z \in \partial D$. Thus

$$
E^{x_{0}} e_{q}\left(\tau_{D}\right)=\int_{\partial D} F\left(x_{0}, z\right) w^{x_{0}}(d z)<\infty
$$

and by Theorem 2.1 (Gauge Theorem) it follows that $E^{x} e_{q}\left(\tau_{D}\right) \simeq 1$ on $D$. Now the equivalence $E^{x} e_{q}\left(\tau_{D}\right) \simeq 1$ means there exist positive constants $c_{1}$ and $c_{2}$ such that $c_{1} \leq \int F(x, z) w^{x}(d z) \leq c_{2}$. Thus for each $x \in \overline{U(a / 2)}$ there is a $z_{1}=z_{1}(x) \in \partial D$ such that $F\left(x, z_{1}\right) \geq c_{1}$. Then by (4.5.2) and (4.5.4) $F(x, z) \geq \tilde{C}_{1}$ for all $z \in \partial D$. Similarly, there is a $z_{2}=z_{2}(x)$ such that $F\left(x, z_{2}\right) \leq c_{2}$. Again by (4.5.2) and (4.5.4), $F(x, z) \leq \tilde{C}_{2}$ for all $z \in \partial D$. Thus there are constants $c_{1}, c_{2}$ such that $c_{1} \leq F(x, z) \leq c_{2},(x, z) \in A_{2}$ and claim (2) is established.

Next it is easy to verify the equivalence

$$
F(x, y) \simeq 1 \quad \text { for }(x, y) \in A_{1} \cup A_{2} .
$$

If $(x, y) \in A_{1}$ hook up $y$ to $y^{\prime} \in \overline{C(a / 8)}$ as before. Then $F(x, y) \simeq F\left(x, y^{\prime}\right) \simeq 1$ since $\left(x, y^{\prime}\right) \in A_{2}$.

From here we can claim

$$
F(x, y) \simeq 1 \quad \text { for }(x, y) \in \overline{U(a)} \times \bar{D} .
$$

By (3) we only need verify (4) for $(x, y) \in A_{3}=\{(x, y) \in \overline{U(a)} \times \overline{U(a / 4)}, 0<$ $|y-x|<r / 2\}$. For such a pair

$$
\begin{aligned}
F(x, y)= & E_{y}^{x}\left[e_{q}\left(\tau_{B(y, r)}\right) F\left(X\left(\tau_{B(y, r)}\right), y\right), \tau_{B(y, r)}<\tau_{D}\right] \\
& +E_{y}^{x}\left[e_{q}\left(\tau_{D}\right) ; \tau_{B(y, r) \backslash\{y\}}=\tau_{D}\right] .
\end{aligned}
$$


But $X\left(\tau_{B(y, r)}\right) \in \overline{U(a / 2)}$ on $\left\{\tau_{B(y, r)}<\tau_{D}, X(0) \in \overline{U(a)}\right\}$ so by $(3), F\left(X\left(\tau_{B(y, r)}\right), y\right)$ $\simeq 1$ and

$$
F(x, y) \simeq E_{y}^{x}\left[e_{q}\left(\tau_{B(y, r) \backslash\{y\}}\right)\right]
$$

and the right side is equivalent to 1 by Lemma 4.3. This proves (4). Finally we establish the assertion of the theorem

$$
F(x, y) \simeq 1 \quad \text { for }(x, y) \in \bar{D} \times \bar{D} .
$$

Note that for $(x, y) \in \overline{C(a)} \times \bar{D}$,

$$
\begin{aligned}
F(x, y)= & E_{y}^{x}\left[e_{q}\left(\tau_{C(a)}\right) F\left(X\left(\tau_{C(a)}\right), y\right), \tau_{C(a)}<\tau_{D}\right] \\
& +E_{y}^{x}\left[e_{q}\left(\tau_{D}\right) ; \tau_{C(a) \backslash\{y\}}=\tau_{D}\right]
\end{aligned}
$$

Since $X\left(\tau_{C(a)}\right) \in \overline{U(a)}$ on $\left\{\tau_{C(a)}<\tau_{D}\right\}$ we can use (4) and conclude that since $F\left(X\left(\tau_{C(a)}\right), y\right) \simeq 1$ one has

$$
F(x, y) \simeq E_{y}^{x}\left[e_{q}\left(\tau_{C(a) \backslash\{y\}}\right)\right] \simeq 1 \text { on } C(a) \times \bar{D} \text {, by our choice of } a .
$$

This together with (4) give (5) and the proof is complete when $x_{0} \in D$. When $x_{0} \in \partial D$ select $r$ so small that $y_{0} \notin B\left(x_{0}, r\right)$ and set

$$
T_{r}=\inf \left\{t>0: X(t) \notin B\left(x_{0}, r\right)\right\} .
$$

Then

$$
F\left(x_{0}, y_{0}\right)=E_{y_{0}}^{x_{0}}\left(e_{q}\left(T_{r}\right) E^{X\left(T_{r}\right)} e_{q}\left(\tau_{D}\right)\right)<\infty
$$

so $E^{X\left(T_{r}\right)} e_{q}\left(\tau_{D}\right)<\infty P_{y_{0}}^{x_{0}}$ a.s. and thus for some $x \in D \cap \partial B\left(x_{0}, r\right), F\left(x, y_{0}\right)<\infty$. The previous part of the proof shows $F \simeq 1$.

THEOREM 4.6. If $F(\cdot) \not \equiv \infty$ then there exist positive constants $c_{1}$ and $c_{2}$ such that $c_{1} \leq F(x, y) \leq c_{2},(x, y) \in \bar{D} \times \bar{D}$.

ProOF. When $E^{x_{0}}\left[e_{q}\left(\tau_{D}\right)\right]<\infty$ for some $x_{0} \in \bar{D}$ it follows from

$$
E^{x_{0}}\left[e_{q}\left(\tau_{D}\right)\right]=\int E_{z}^{x_{0}}\left[e_{q}\left(\tau_{D}\right)\right] w^{x_{0}}(d z)
$$

that $E_{z}^{x_{0}}\left[e_{q}\left(\tau_{D}\right)\right]<\infty$ for $w^{x}$ a.e. $z$ and thus Theorem 4.2 implies the desired conclusion.

Part (4.7.2) of the next result was proven in [30] with $A=\Delta / 2, q \in K_{d}(D), D$ a $C^{1,1}$ domain.

THEOREM 4.7. If $F(\cdot) \not \equiv \infty$ then

$$
w^{x}(d z)=F(x, z) w^{x}(d z)
$$

and for $x, y \in D, x \neq y$,

$$
\begin{aligned}
& G_{L}(x, y)=F(x, y) G(x, y) \\
& G_{L}(x, y)=G(x, y)-\int_{D} G_{L}(x, w) q(w) G(w, y) d w \\
& E_{y}^{x} e_{q}\left(\tau_{D}\right)=1-G(x, y)^{-1} \int_{D} G_{L}(x, w) q(w) G(w, y) d w .
\end{aligned}
$$


PROOF. Formula (4.7.1) is a direct consequence of the Feynman-Kac formula. In order to establish (4.7.2)-(4.7.4) write

$$
e_{q}\left(\tau_{D}\right)=1-\int_{0}^{\tau_{D}} q(X(s)) \exp \left\{-\int_{s}^{\tau_{D}} q(X(t)) d t\right\} d s
$$

Then on taking expectations,

$$
\begin{aligned}
F(x, y) & =1-E_{y}^{x} \int_{0}^{\tau_{D}} q(X(s)) \exp \left\{-\int_{s}^{\tau_{D}} q(X(t)) d t\right\} d s \\
& =1-E_{y}^{x} \int_{0}^{\tau_{D}} q(X(s)) E_{y}^{x}\left[\exp \left\{-\int_{s}^{\tau_{D}} q(X(t)) d t\right\} \mid X(s)\right] d s \\
& =1-E_{y}^{x} \int_{0}^{\tau_{D}} q(X(s)) E_{y}^{x}\left[\exp \left\{-\int_{s}^{\tau_{D}} q(X(t)) d t\right\}\right] d s .
\end{aligned}
$$

Now, $F(X(s), y)$ is bounded so by assumption that $q \in K_{d}$ and the 3G Theorem we can interchange the order of integration in the last line. This results in

$$
\begin{aligned}
F(x, y) & =1-G(x, y)^{-1} \int_{D} G(x, w) q(w) F(w, y) G(w, y) d w \\
& =1-G(x, y)^{-1} \int_{D} G(w, x) q(w) F(y, w) G(y, w) d w
\end{aligned}
$$

which follows by the symmetry of $G$ and the fact that the diffusion $X$ started at $x$ conditioned to hit $y$ when run backwards is the diffusion $X$ started at $y$ conditioned to hit $x$. Thus

$$
F(x, y) G(x, y)=G(x, y)-\int_{D} F(y, w) G(y, w) q(w) G(w, x) d w .
$$

Since the term on the left and the first term on the right are symmetric in $x$ and $y$ so is the integral term and hence

$$
F(x, y) G(x, y)=G(x, y)-\int_{D} F(x, w) G(x, w) q(w) G(w, y) d w
$$

Now apply the operator $A$ in the variable $y$ to both sides. On the right, one gets $\delta_{x}(d y)-F(x, y) G(x, y) q(y)$. Consequently, again acting in the variable $y$, $L F(x, y) G(x, y)=\delta_{x}(d y)$ and this will establish (4.7.2) once we verify $F(x, y) G(x, y)$ $\rightarrow 0$ as $y \rightarrow z \in \partial D$, for $z$ a regular point. In fact, this happens for all $z \in \partial D$ since all points on $\partial D$ are regular for the Dirichlet problem for $A$ by results of [23] and the boundedness of $F(x, y)$. Formula (4.7.3) and (4.7.4) now follow easily.

THEOREM 4.8. Assume $F(\cdot) \not \equiv \infty$. Then there exist positive constants $c_{1}$ and $c_{2}$ such that

$$
\begin{aligned}
& c_{1} w^{x}(E) \leq w_{L}^{x}(E) \leq c_{2} w^{x}(E), \quad E \in B(\partial D), x \in D . \\
& c_{1} G(x, y) \leq G_{L}(x, y) \leq c_{2} G(x, y), \quad x, y \in D .
\end{aligned}
$$

PROOF. This follows immediately from Theorems 4.2 and 4.7.

THEOREM 4.9. Assume $F(\cdot) \not \equiv \infty$. Then $F(x, y)=E_{y}^{x} e_{q}\left(\tau_{D}\right)$ is a continuous function on $\bar{D} \times \bar{D}$.

PROOF. Using Harnack's inequality, Theorem 5.1, and classical arguments one can prove $G_{L}(x, y)$ is jointly continuous on $\bar{D} \times \bar{D} \backslash \operatorname{diag}(\bar{D} \times \bar{D})$. The same is 
true for $G(x, y)$. This gives, together with (4.7.4), the joint continuity of $F(x, y)$ on $D \times D \backslash \operatorname{diag}(D \times D)$. For this one also uses the $3 \mathrm{G}$ Theorem to apply dominated convergence and the fact that

$$
G(x, y)^{-1} G_{L}(x, w) G(w, y) \rightarrow G\left(x^{\prime}, y^{\prime}\right)^{-1} G_{L}\left(x^{\prime}, w\right) G\left(w, y^{\prime}\right)
$$

as $x \rightarrow x^{\prime}, y \rightarrow y^{\prime}$ for a.e. $w \in D$, for $x^{\prime}, y^{\prime} \in D, x^{\prime} \neq y^{\prime}$. For $x^{\prime} \in D, y^{\prime} \in \partial D$ and $x \rightarrow x^{\prime}, y \rightarrow y^{\prime}, y \in D$ one can use the boundary Harnack principle and maximum principle for $A$ to conclude $G(w, y) / G(x, y) \rightarrow K\left(w, y^{\prime}\right) / K\left(x^{\prime}, y^{\prime}\right)$ where $K\left(w, y^{\prime}\right)$ and $K\left(x^{\prime}, y^{\prime}\right)$ are even continuous (in fact Hölder) as a function of $y^{\prime} \in \partial D$. Thus applying dominated convergence with the aid of the $3 \mathrm{G}$ Theorem one obtains

$$
\begin{aligned}
\lim _{\substack{x \rightarrow x^{\prime} \\
y \rightarrow y^{\prime}}} F(x, y) & =1-\lim _{\substack{x \rightarrow x^{\prime} \\
y \rightarrow y^{\prime}}} \int_{D} \frac{G_{L}(x, w) q(w) G(w, y)}{G(x, y)} d w \\
& =1-\int_{D} \frac{G_{L}\left(x^{\prime}, w\right) q(w) K\left(w, y^{\prime}\right)}{K\left(x^{\prime}, y^{\prime}\right)} d w \\
& =F\left(x^{\prime}, y^{\prime}\right)
\end{aligned}
$$

and $F$ is continuous on $D \times \bar{D} \backslash \operatorname{diag}(D \times \bar{D})$. Since $F(x, y)$ is symmetric in $x$ and $y$ it follows that $F$ is continuous on $\bar{D} \times D \backslash \operatorname{diag}(\bar{D} \times D)$ as well. Now suppose $x^{\prime}$, $y^{\prime} \in \bar{D}$ and select $r>0$ so that $\left|x^{\prime}-y^{\prime}\right| \geq 3 r$. Next let $x, y \in \bar{D}, x \rightarrow x^{\prime}, y \rightarrow y^{\prime}$ and assume $\left|x^{\prime}-x\right|<r / 2$ and $\left|y-y^{\prime}\right|<r / 2$. Then, defining $D_{1}=D \backslash B\left(x^{\prime}, r\right)$, $D_{2}=D \backslash B\left(y^{\prime}, r\right)$, we have, writing $D_{1}^{c}=D \backslash D_{1}$,

$$
\begin{aligned}
E_{y}^{x}\left[e_{q}\left(\tau_{D}\right)\right]= & \left.E_{y}^{x}\left[e_{q}\left(\tau_{D_{1}^{c}}\right)\right] e_{q}\left(\theta_{\tau D_{1}^{c}} \cdot \tau_{D_{2}}\right) e_{q}\left(\theta_{\tau_{D_{2}}} \cdot \tau_{D}\right)\right] \\
= & \int_{\partial D_{1} \cap D} \int_{\partial D_{2} \cap D}{ }_{1} E_{z}^{x}\left[e_{q}\left(\tau_{D_{1}^{c}}\right)\right]_{2} E_{u}^{z}\left[e_{q}\left(\tau_{D_{2}}\right)\right] E_{y}^{u}\left[e_{q}\left(\tau_{D}\right)\right] \\
\cdot & P_{y}^{x}\left(X\left(\tau_{D_{1}^{c}}\right) \in d z\right) P_{y}^{z}\left(X\left(\tau_{D_{2}}\right) \in d u\right) .
\end{aligned}
$$

The measure ${ }_{1} P_{z}^{x}$ is on paths started at $x$ conditioned to exit $D_{1}^{c}$ at $z$ and the measure ${ }_{2} P_{u}^{z}$ is on paths started at $z$ conditioned to leave $D_{2}$ at $u$. Now by part of our previous argument for the conditional gauge in $D_{11} E_{z}^{x}\left[e_{q}\left(\tau_{D_{1}^{c}}\right)\right]$ is continuous on $\overline{B\left(x^{\prime}, r / 2\right) \cap D} \times \partial D_{1} \cap D$ and ${ }_{2} E_{y}^{u}\left[e_{q}\left(\tau_{D}\right)\right]$ is continuous on $\partial D_{2} \cap D \times \overline{B\left(y^{\prime}, r / 2\right) \cap D}$. Expressions can also be worked out for $P_{y}^{x}\left(X\left(\tau_{D_{1}^{c}}\right) \in d z\right)$ and $P_{y}^{z}\left(X\left(\tau_{D_{2}}\right) \in d u\right)$. For the first, let ${ }_{1} w^{x}(d z)$ denote harmonic measure for $A$ on $D_{1}^{c},{ }_{1} K(x, z)$ the Martin kernel for $A$ on $D_{1}^{c}$ normalized so that ${ }_{1} K\left(x_{0}, z\right) \equiv 1$ for some $x_{0} \in D_{1}^{c}$. Then

$$
P_{y}^{x}\left(X\left(\tau_{D_{1}^{c}}\right) \in d z\right)=\frac{G\left(x_{0}, y\right)_{1} K(x, z)}{G(x, y)}{ }_{1} \omega^{x_{0}}(d z) \text {. }
$$

Similarly, letting ${ }_{2} w^{z}(d u)$ and ${ }_{2} K(z, u)$ denote harmonic measure and the Martin kernel, normalized so that ${ }_{2} K\left(z_{0}, u\right)=1$ for some $z_{0} \in D_{2}$, for $A$ we also have

$$
P_{y}^{z}\left(X\left(\tau_{D_{2}}\right) \in d u\right)=\frac{G\left(z_{0}, y\right){ }_{2} K(z, u)}{G(z, y)}{ }_{2} w^{z_{0}}(d u)
$$

Thus,

$$
\begin{aligned}
& P_{y}^{x}\left(X\left(\tau_{D_{1}^{c}}\right) \in d z\right) P_{y}^{z}\left(X\left(\tau_{D_{2}}\right) \in d u\right) \\
& \quad=\frac{G\left(x_{0}, y\right){ }_{1} K(x, z) G\left(z_{0}, y\right)_{2} K(z, u)}{G(x, y) G(z, y)}{ }_{1} w^{x_{0}}(d z){ }_{2} w^{z_{0}}(d u) .
\end{aligned}
$$


However, $G\left(x_{0}, y\right)_{1} K(x, z) / G(x, y)$ is continuous for $(x, y) \in \overline{B\left(x^{\prime}, r / 2\right)} \times \overline{B\left(y^{\prime}, r / 2\right)}$ for $z \in \partial D_{1} \cap D$ as the boundary Harnack and maximum principles imply. Similarly, $G\left(z_{0}, y\right)_{2} K(z, u) / G(z, y)$ is continuous for $y \in \overline{B\left(y^{\prime}, r / 2\right)}$ when $z \in \partial D_{1} \cap D$. Thus by the boundedness of all the gauges in (4.9.1) it follows that $F(x, y)$ is continuous on $\overline{B\left(x^{\prime}, r / 2\right)} \times \overline{B\left(y^{\prime}, r / 2\right)}$ and thus on $\bar{D} \times \bar{D} \backslash \operatorname{diag}(\bar{D} \times \bar{D})$. To obtain continuity on all of $\bar{D} \times \bar{D}$ note that $F(x, x)=1$ for all $x \in \bar{D}$ so $F$ is continuous on $\operatorname{diag} \bar{D} \times \bar{D}$.

The last case to consider is $x^{\prime} \in \partial D, x, y \in \bar{D}, x \rightarrow x^{\prime}, y \rightarrow y^{\prime}$. We may ignore the case $x=y$ since then $F(x, y)=1$ and $F\left(x^{\prime}, x^{\prime}\right)=1$. Set

$$
K(x, w, y)=G(x, y)^{-1} G(x, w) G(w, y)
$$

and note that $K$ extends continuously in $x$ and $y$ up to $\partial D$ as long as $x \neq y$. We claim $K(x, w, y) \rightarrow 0$ for a.e. $w \in D$ as $x, y \rightarrow x^{\prime}$. Assume $\delta(x)<\delta(y)$ by symmetry. If $|x-y| \leq \delta(x) / 2$ then by (2.3.2) it is easily seen that $K(x, w, y) \rightarrow 0$. If $|x-y|>\delta(y) / 2$, let $r=|x-y|$ and observe that $\delta(x) \vee \delta(y)<2 r$. If $\delta(x) \geq r / 3$ and $\delta(y) \geq r / 3$ select a point $\hat{x} \in \partial B(y, r / 6)$. By constructing a Harnack chain of length independent of $r$ from $x$ to $\hat{x}$ in $D \backslash B(y, r / 12) \cup\{w\}$ (we assume $w$ is far away) it follows that $K(x, w, y) \simeq K(\hat{x}, w, y)$. But $|\hat{x}-y|=r / 6<\delta(y) / 2$ and $K(\hat{x}, w, y) \rightarrow 0$ by the first part of the argument. If $\delta(x)<r / 3$ and $\delta(y)>r / 3$, let $x^{*}$ be the closest point to $x$ on $\partial D$ and $A_{r / 3}\left(x^{*}\right)$ as defined following Theorem 3.1. Then $K\left(A_{r / 3}\left(x^{*}\right), w, y\right) \simeq K(x, w, y)$ by the boundary Harnack principle and again we construct a Harnack chain whose length is independent of $r$ from $A_{r}\left(x^{*}\right)$ to $\hat{x}$ to obtain $K(x, w, y) \simeq K(\hat{x}, w, y)$ which tends to 0 as before. The last case $\delta(x)<r / 3, \delta(y)<r / 3$ is similar, $K(x, w, y) \simeq K\left(A_{r}\left(x^{*}\right), w, A_{r}\left(y^{*}\right)\right)$ and now make a Harnack chain from $A_{r}\left(x^{*}\right)$ to a point $\hat{x} \in \partial B\left(A_{r}\left(y^{*}\right), r / 6\right)$ in $D \backslash B\left(A_{r}\left(y^{*}\right), r / 12\right)$ whose length is independent of $r$ to conclude $K(x, w, y) \simeq K\left(\hat{x}, w, A r\left(y^{*}\right)\right)$ and this tends to 0 as $r$ tends to 0 . The proof is concluded by the 3G Theorem, dominated convergence and the now established claim that $K(x, w, y) \rightarrow 0$ a.e. $w$ as $x, y \rightarrow x^{\prime}$.

5. Potential theory for $L$. Throughout this section we make the assumption that $F\left(x_{0}, y_{0}\right)<\infty$ for some $x_{0} \neq y_{0}, x_{0}, y_{0} \in \bar{D}$. Equivalent assumptions are given in $\S 2.5$. One such is that $F(\cdot) \not \equiv \infty$.

Theorem 4.9 coupled with the CGT (Conditional Gauge Theorem) give some quick results on $w_{L}$ and $G_{L}$. For example, from (4.9.1) it follows that under our assumption, $w_{L}^{x}$ and $w^{x}$ are mutually absolutely continuous with the Radon-Nikodym derivative, either way, bounded above and below. Recently, in [15 and 18], conditions on $A$ were given implying the absolute continuity of $w^{x}$ with respect to surface area $\sigma$. Quite trivially these same conditions on $A$ imply the absolute continuity of $w_{L}^{x}$ with respect to $\sigma$. Also, one gets $A_{p}$-weight conditions simultaneously on $w^{x}$ and $w_{L}^{x}$ with respect to $\sigma$ and consequently such results as unique solvability of the Dirichlet problem $L u=0$ in $D, u=\phi$ on $\partial D$ for $\phi \in L^{p}(d \sigma)$. We refer the reader to [15 and 18] for the exact results one can get here for $L$ from the corresponding results for $A$.

Similarly, one sees from (4.9.2) and the CGT that $G_{L}$ is a bounded function times $G$. This implies for example that the $L$-potential and $A$-potential of any measure $\mu$ with $\operatorname{supp} \mu \subseteq D$ are equivalent in that the two-sided inequality $c^{-1} G_{L} \mu(x) \leq$ $G \mu(x) \leq c G_{L} \mu(x)$ holds. One consequence of this is that the $L$-capacity of a set $B$, $c_{L}(B)$, is equivalent to its $A$-capacity, $c(B)$, i.e. $c^{-1} c_{L}(B) \leq c(B) \leq c c_{L}(B)$. These 
equivalences say that the fine topology, polar sets etc. are the same for $A$ and $L$. This was also observed in [6] using perturbation of harmonic spaces. We next consider Harnack's principle. [1] considered this for $A=\Delta / 2, D$ a ball, $q \in K_{d}$. Chiarenza et al. [9] have it under the same assumptions we make here with different techniques.

THEOREM 5.1 (HARNACK'S PRINCIPLE). If $F(\cdot) \not \equiv \infty$ there exists a positive constant $c$, such that for every $u \in \mathcal{L}^{+}(D)$ and for any ball $B(z, 2 r)=B_{2 r} \subset D$ with $r<r_{0}$

$$
u(x) \leq c u(y) \quad \text { for } x, y \in B(z, r)=B_{r} .
$$

ProOF. The Harnack principle holds for $A$ by [24]. Applying the CGT in $B_{2 r}$ for $x, y \in B_{r}$

$$
\begin{aligned}
u(x) & =\int_{\partial B_{2 r}} u(z) F(x, z) w^{x}(d z) \\
& \leq c \int_{\partial B_{2 r}} u(z) w^{x}(d z) \quad \text { by CGT with } c \text { independent of } r \\
& \leq c \int_{\partial B_{2 r}} u(z) w^{y}(d y) \quad \text { by Moser's Harnack principle for } A \\
& \leq c \int_{\partial B_{2 r}} u(z) F(y, z) w^{y}(d z) \quad \text { by CGT } \\
& =c u(y) .
\end{aligned}
$$

In a similar manner one gets the boundary Harnack principle.

THEOREM 5.2 (BOUNDARY HARNACK PRINCIPLE). If $F(\cdot) \not \equiv \infty$, there exists a positive constant $c$, such that for any $u, v \in \mathcal{L}^{+}(D)$ and $z \in \partial D, r<r_{0}$, with $u$ and $v$ vanishing continuously on $B(z, 2 r) \cap \partial D$, we have

$$
\frac{u}{v}(x) \leq c \frac{u}{v}(y), \quad x, y \in B(z, r) \cap D .
$$

PROOF. Assume $D_{r}=B(z, 3 r / 2) \cap D$ has the same Lipschitz character as $D$. If this is not true we could take a domain $D_{r}$ with the same Lipschitz character as $D$ and $D \cap B(z, r) \subset D_{r} \subset D \cap B(z, 3 r / 2)$. Write $w_{r}^{x}, w_{L, r}^{x}$ for $A$-harmonic measure on $D_{r}$ and $L$-harmonic measure on $D_{r}$, respectively. Then $w_{r}^{x} \simeq w_{L, r}^{x}$ and the constants in this equivalence can be taken as the same constants for the analogous statement on $D$. If $u$ and $v$ are as in the theorem let $\hat{u}$ and $\hat{v}$ be the $A$-harmonic functions on $D_{r}$ whose boundary values are $u$ and $v$, respectively. Then $u \simeq \hat{u}$ and $v \simeq \hat{v}$ on $D_{r}$ and since

$$
\frac{\hat{u}}{\hat{v}}(x) \leq c \frac{\hat{u}}{\hat{v}}(y) \quad \text { for } x, y \in B(z, r) \cap D
$$

it follows that

$$
\frac{u}{v}(x) \leq c \frac{u}{v}(y), \quad x, y \in B(z, r) \cap D .
$$

The BHP says functions in $\mathcal{L}^{+}(D)$ vanishing on a portion of $\partial D$ vanish at the same rate on a subportion. Considering the Feynman-Kac formula $u(x)=$ $E_{x}\left\{f\left(X\left(\tau_{D^{-}}\right)\right) e_{q}\left(\tau_{D}\right)\right\}$ for solutions $L u=0$ and $u=f$ on $\partial D$ and the stochastic representation for solutions to $A v=0, v=f$ on $\partial D, v(x)=E_{x}\left\{f\left(X\left(\tau_{D^{-}}\right)\right)\right\}$one might expect that $e_{q}\left(\tau_{D}\right) \rightarrow 1$ nicely enough as $x \rightarrow \partial D$ so that $u$ and $v$ should vanish at the same rate at $\partial D$. This is in fact the case. 
THEOREM 5.3 (COMPARISON OF SOlutions). Assume $F(\cdot) \not \equiv \infty$. Then there exist positive constants $c$ and $r_{0}$ such that if $u \in \mathcal{L}^{+}(D), v \in A^{+}(D)$ vanish continuously on $B(z, 2 r) \cap \partial D$, with $r<r_{0}$ and $z \in \partial D$, then

$$
\frac{u}{v}(x) \leq c \frac{u}{v}(y), \quad x, y \in B(z, r) \cap D .
$$

ProOF. The CGT holds in $B(z, 2 r) \cap D$ and the constant $c$ does not change as the Lipschitz character of $B(z, 2 r) \cap D$ is essentially the same as for $D$. This is almost true but can easily be taken care of. Let $w_{r}^{x}$ be harmonic measure for $A$ on $B(z, 3 r / 2) \cap D$. Using this and the BHP for $A^{+}(D)$, for $x, y \in B(z, r) \cap D$

$$
\begin{aligned}
\frac{u}{v}(x) & \leq c \int_{\partial(B(z, 3 r / 2) \cap D)} u(y) w_{r}^{x}(d y) / v(x) \\
& \leq c \int_{\partial(B(z, 3 r / 2) \cap D)} u(z) w_{r}^{y}(d z) / v(y) \quad \text { by BHP for } \AA^{+}(D) \\
& \leq c(u / v)(y) \quad \text { by CGT on } B(z, 3 r / 2) \cap D . \quad \square
\end{aligned}
$$

We can refine Theorem 5.3 by asking whether $\lim _{x \rightarrow z}(u / v)(x)$ exists for $z \in$ $B(z, r) \cap \partial D$. The answer is yes and is contained in

THEOREM 5.4. Suppose $F(\cdot) \not \equiv \infty$. Then

(5.4.1) $\lim _{x \rightarrow z} G_{L}(x, y) / G(x, y)$ exists for every $z \in \partial D, x \in D$. Also, for $u$ and $v$ as in Theorem 5.3

(5.4.2) $\lim _{x \rightarrow w}(u / v)(x)$ exists for every $w \in \partial D \cap B(z, r)$. In fact, if $q_{1}, q_{2} \in$ $K_{d}(D)$ have finite gauges and $\left(-A+q_{1}\right) u=0$ on $D,\left(-A+q_{2}\right) v=0$ on $D$ with $u, v$ positive solutions vanishing continuously on $\partial D \cap B(z, 2 r)$ then

(5.4.3) $\lim _{x \rightarrow w}(u / v)(x)$ exists for every $w \in \partial D \cap B(z, r)$. In fact, $G_{L}(\cdot) / G(\cdot)$ extends to a continuous function on $\bar{D} \times \bar{D}$ with value 1 on the diagonal. The ratios $u / v$ in (5.4.2) and (5.4.3) extend to continuous functions on $\partial D \cap B(z, r)$.

ProOF. From the selfadjointness of $A$ and formula (4.8.3)

$$
\begin{aligned}
\frac{G_{L}(x, y)}{G(x, y)} & =\frac{G_{L}(y, x)}{G(y, x)}=1-G(y, x)^{-1} \int_{D} G_{L}(y, w) q(w) G(w, x) d w \\
& =1-\int_{D} \frac{G_{L}(y, w) G(w, x)}{G(y, x)} q(w) d w .
\end{aligned}
$$

Using the estimate $G_{L}(y, w) \leq c G(y, w)$ and the 3G Theorem, since $q \in K_{d}(D)$, dominated convergence can be applied to get for $z \in \partial D$

$$
\begin{aligned}
\lim _{x \rightarrow z} \frac{G_{L}(x, y)}{G(x, y)} & =1-\int_{D} G_{L}(y, w)\left[\lim _{x \rightarrow z} \frac{G(w, x)}{G(y, x)}\right] q(w) d w \\
& =1-\int_{D} G_{L}(y, w) \frac{K(w, z)}{K(y, z)} q(w) d w .
\end{aligned}
$$

Notice this last is $E_{z}^{y} e_{q}\left(\tau_{D}\right)$ and is continuous in $z \in \partial D$, by Theorem 4.2. Similarly, with $x \in D$ fixed, $G_{L}(x, \cdot) / G(x, \cdot)$ extends to a continuous function on $\bar{D} \backslash\{x\}$. Now let $x \rightarrow w$ and $y \rightarrow w$ with $w \in D$. Then

$$
\lim _{x, y \rightarrow w} \frac{G_{L}(x, y)}{G(x, y)}=1-\lim _{x, y \rightarrow w} G(x, y)^{-1} \int_{D} G_{L}(x, u) q(u) G(u, y) d u .
$$


For $u \neq w, G(x, y)^{-1} G_{L}(x, u) G(u, y) \rightarrow 0$ as $x, y \rightarrow w$. Applying the 3G Theorem, $q \in K_{d}(D)$ and dominated convergence we conclude

$$
\lim _{x, y \rightarrow w} G(x, y)^{-1} \int_{D} G_{L}(x, u) q(u) G(u, y) d u=0
$$

and $G_{L}(x, y) / G(x, y)$ is continuous on the set $D \times \bar{D} \cup \bar{D} \times D$.

Suppose now $u$ and $v$ are as in (5.4.2). Then

$$
\lim _{x \rightarrow z} \frac{u(x)}{v(z)}=\lim _{x \rightarrow z} \frac{u(x)}{G(x, y)} \cdot \frac{G_{L}(x, y)}{v(x)} \cdot \frac{G(x, y)}{G_{L}(x, y)} .
$$

We have just shown $\lim _{x \rightarrow z} G(x, y) / G_{L}(x, y)$ exists and is a continuous function of $z \in \partial D$. The other two $\operatorname{limits}_{1} \lim _{x \rightarrow z} u(x) / G(x, y)$ and $\lim _{x \rightarrow z} G_{L}(x, y) / v(x)$, exist and are continuous (even Hölder). This follows since the boundary Harnack principle and weak maximum principle hold for both $A$ and $L$ and what are by now standard arguments work (see either [22 or 2]). Part (5.4.3) follows easily from (5.4.2).

Now one feature of the boundary Harnack principle is that coupled with the weak maximum principle (it is easy to verify $L$ satisfies the weak maximum principle) one gets the Martin boundary of $D$ to be equal to the Euclidean boundary of $D$. Such arguments are found in [22].

TheOREM 5.5 (MARTin REPRESENTATiON). Assume $F(\cdot) \not \equiv \infty$. The Martin boundary for $L$ on $D$ is the Euclidean boundary $\partial D$. Every $u \in \mathcal{L}^{+}$has the unique representation

$$
u(x)=\int_{\partial D} K_{L}(x, z) \mu(d z)
$$

for some Borel measure $\mu$ on $\partial D$ where

$$
K_{L}(x, z)=\frac{F(1 ; x, z)}{F\left(1 ; \tilde{x}_{0}, z\right)} K(x, z)
$$

where we have made the normalization $K\left(\tilde{x}_{0}, \cdot\right) \equiv 1$.

Another consequence of the CGT is that once the gauge is not identically infinite, the set of regular points on $\partial D$ for the Dirichlet problems for $A$ and $L$ are identical. There are several ways to establish this. According to a theorem of Brelot, the points on the Martin boundary are regular for the Dirichlet problem. Theorem 5.5 shows the Martin boundary coincides with the Euclidean boundary for the operators $L$ and $A$. One might also consider applying Wiener's test and observing, since $G_{A}$ and $G_{L}$ are bounded multiples of one another, that the $A$-capacity of any set is equivalent to its $L$-capacity. Finally, by [23] the regular points on $\partial D$ are the same for $A$ and $\Delta$. Thus we have the following.

THEOREM 5.6 (REgulaRity OF BOUNDARY POINTS). If $F(\cdot) \not \equiv \infty$ then all points on $\partial D$ are regular for the Dirichlet problem for $L$ when $D$ is Lipschitz.

The existence of nontangential limits a.e. with respect to surface measure for $u \in \mathcal{L}^{+}(\Sigma)$ where $\Sigma=B(0,1)$ follows from the CGT and results of [9]. More explicitly define, for $0<\sigma<1$ and $z \in \partial \Sigma, \Gamma_{\sigma}(z)$ to be the convex hull of $B(0, \sigma)$ 
and $\{z\}$. The maximal function of $u$ is $u^{*}(z)=\max _{\Gamma_{\sigma}(z)} u$. Define for $\nu$ a positive Borel measure on $\partial \Sigma$, with $\nu(\partial \Sigma)<\infty$, the Hardy-Littlewood maximal operator

$$
\mathcal{M}_{\mu}(\nu)(z)=\sup _{r>0} \frac{\nu(\Delta(z, r))}{\mu(\Delta(z, r))}
$$

where $\mu$ is a fixed positive finite Borel measure on $\partial \Sigma$.

THEOREM 5.7. Assume $F(\cdot) \not \equiv \infty$. Let $\nu$ be a positive finite Borel measure on $\partial \Sigma$. Let $u_{L}(x)=\int K_{L}(x, z) d \nu(z)$ where $K_{L}$ is as in Theorem 5.5 with $D=\Sigma$. Then there exists a positive $c=c(\lambda, q, d)$ such that $u_{L}^{*}(z) \leq c \mathcal{M}_{w_{L}^{0}}(\nu)(z)$.

ProOF. The CGT implies $\mathcal{M}_{w_{L}^{0}}(\nu) \simeq \mathcal{M}_{w^{0}}(\nu)$ and $u_{A}^{*} \simeq u_{L}^{*}$ where $u_{A}(x)=$ $\int K(x, z) d \nu(z)$. Thus Theorem 5.6 follows from Theorem 4.3 of [9] which says $u_{A}^{*}(z) \leq c \mathcal{M}_{w^{0}}(\nu)(z)$.

The CGT allows a modification of the proofs of Theorems 4.4 and 4.5 in [9] to give the following result.

THEOREM 5.8. Suppose $F(\cdot) \not \equiv \infty$ and $u \in \mathcal{L}^{+}(\Sigma)$. Then there exists a set $E \cap \partial \Sigma$ with $w_{L}^{0}(E)=0$ (and therefore $w^{0}(E)=0$ ) such that $\lim _{y \rightarrow z ; y \in \Gamma_{\sigma}}(z) u(y)$ exists for each $z \in E^{c}$ and every $0<\sigma<1$.

THEOREM 5.9. Assume $F(\cdot) \not \equiv \infty$ and $u$ is a bounded solution to $L u=0$ in $\Sigma$. Then there exists an $f \in L^{\infty}\left(\partial \Sigma, d w_{L}^{0}\right)$ such that

$$
u(x)=\int_{\partial \Sigma} f(z) K(x, z) d w_{L}^{0}(z),
$$

where we have normalized so that $K(0, \cdot) \equiv 1$.

\section{REFERENCES}

1. M. Aizenman and B. Simon, Brownian motion and Harnack inequality for Schrödinger operators, Comm. Pure Appl. Math. 35 (1982), 209-273.

2. M. Anderson and R. Schoen, Positive harmonic functions on complete manifolds of negative curvature, preprint.

3. D. Aronson, Non-negative solutions of linear parabolic equations, Ann. Sci. Norm. Sup. Pisa 23 (1968).

4. R. Bañuelos, On an estimate of Cranston and McConnell concerning the lifetime of certain diffusions, preprint.

5. R. Blumenthal and R. Getoor, Markov processes and potential theory, Academic Press, New York, 1968.

6. A. Boukricha, W. Hansen and H. Hueber, Continuous solutions of the generalized Schrödinger equation and perturbation of harmonic spaces, preprint.

7. J. Brossard, Le problème de Dirichlet pour l'opérateur de Schrödinger, preprint.

8. L. Caffarelli, E. Fabes, S. Mortola and S. Salsa, Boundary behavior of nonnegative solutions of elliptic operators in divergence form, Indiana J. Math. 30 (1981), 621-640.

9. F. Chiarenza, E. Fabes and N. Garofalo, Harnack's inequality for Schrödinger operators and the continuity of solutions, Proc. Amer. Math. Soc. 98 (1986), 415-425.

10. K. L. Chung, The gauge and conditional gauge theorem, Séminaire de Probabilitiés XIX, Lecture Notes in Math., vol. 1123, Springer, 1983/84, pp. 496-503.

11. K. L. Chung, P. Li and R. J. Williams, Comparison of probability and classical methods for the Schrödinger equation, Exposition. Math. 4 (1986), 271-278.

12. K. L. Chung and M. Rao, Feynman-Kac functional and Schrödinger equation, Sem. Stoch. Proc., Birkhäuser, Boston, Mass., 1981.

13. K. L. Chung and Z. Zhao, From Brownian motion to the Schrödinger equation, preprint. 
14. M. Cranston, Lifetime of conditioned Brownian motion in Lipschitz domains, Z. Wahrsch. Verw. Gebiete 70 (1985), 335-340.

15. B. E. J. Dahlberg, On the absolute continuity of elliptic measures, preprint.

16. E. DiGiorgi, Sulla differentiabilitá l'analizitá degli integrali multipli regolari, Mem. Acad. Sci. Torino, S. III 1 (1957), 25-43.

17. J. L. Doob, Conditioned Brownian motion and the boundary limits of harmonic functions, Bull. Soc. Math. France 85 (1957), 431-458.

18. E. Fabes, D. Jerison and C. Kenig, Necessary and sufficient conditions for absolute continuity of elliptic-harmonic measure, Ann. of Math. (2) 119 (1984), 121-141.

19. N. Falkner, Feynman-Kac functionals and positive solutions of $\frac{1}{2} \Delta u+q u=0, \mathrm{Z}$. Wahrsch. Verw. Gebiete. 65 (1983), 19-31.

20. Conditional Brownian motion in rapidly exhaustible domains, preprint.

21. M. Fukushima, Dirichlet forms and Markov processes, North-Holland/Kodauzha, 1980.

22. D. Jerison and C. Kenig, Boundary behavior of harmonic functions in nontangentially accessible domains, Ann. of Math. (2) 113 (1981), 367-382.

23. W. Littman, G. Stampacchia and H. F. Weinberger, Regular points for elliptic equations with discontinuous coefficients, Ann. Scuola Norm. Sup. Pisa (3) 17 (1963), 45-79.

24. J. Moser, On Harnack's inequality for elliptic differential equations, Amer. J. Math. 80 (1958), 931-954.

25. J. Nash, Continuity of the solutions of parabolic and elliptic equations, Amer. J. Math. 80 (1958), 931-954.

26. T. Salisbury, A Martin boundary in the plane, Trans. Amer. Math. Soc. 293 (1986), 623-642.

27. B. Simon, Schrödinger semigroups, Bull. Amer. Math. Soc. 7 (1982), 447-526.

28. Z. Zhao, Conditional gauge and unbounded potential, Z. Wahrsch. Verw. Gebiete. 65 (1983), 13-18.

29. __ Uniform boundedness of conditional gauge and Schrödinger equations, Comm. Math. Phys. 93 (1984), 19-31.

30. __ Green function for Schrödinger operator and conditioned Feyman-Kac gauge, J. Math. Anal. Appl. 116 (1986), 309-334.

Department of Mathematics, University of Rochester, Rochester, New YORK 14608

Department of Mathematics, University of Minnesota, Minneapolis, MinNESOTA 55455

Department of Mathematics, University of Missouri, Columbia, Missouri 65211 